\title{
Gravity from entanglement and RG flow in a top-down approach
}

\author{
O-Kab Kwon, ${ }^{a}$ Dongmin Jang, ${ }^{a}$ Yoonbai $\mathrm{Kim}^{a}$ and D.D. Tolla ${ }^{a, b}$ \\ ${ }^{a}$ Department of Physics, BK21 Physics Research Division, \\ Institute of Basic Science, Sungkyunkwan University, \\ Suwon 440-746, South Korea \\ ${ }^{b}$ University College, Sungkyunkwan University, \\ Suwon 440-746, South Korea \\ E-mail: okab@skku.edu, dongmin@skku.edu, yoonbai@skku.edu, \\ ddtolla@skku.edu
}

ABSTRACT: The duality between a $d$-dimensional conformal field theory with relevant deformation and a gravity theory on an asymptotically $\mathrm{AdS}_{d+1}$ geometry, has become a suitable tool in the investigation of the emergence of gravity from quantum entanglement in field theory. Recently, we have tested the duality between the mass-deformed ABJM theory and asymptotically $\mathrm{AdS}_{4}$ gravity theory, which is obtained from the KK reduction of the 11-dimensional supergravity on the LLM geometry. In this paper, we extend the KK reduction procedure beyond the linear order and establish non-trivial KK maps between 4-dimensional fields and 11-dimensional fluctuations. We rely on this gauge/gravity duality to calculate the entanglement entropy by using the Ryu-Takayanagi holographic formula and the path integral method developed by Faulkner. We show that the entanglement entropies obtained using these two methods agree when the asymptotically $\mathrm{AdS}_{4}$ metric satisfies the linearized Einstein equation with nonvanishing energy-momentum tensor for two scalar fields. These scalar fields encode the information of the relevant deformation of the ABJM theory. This confirms that the asymptotic limit of LLM geometry is the emergent gravity of the quantum entanglement in the mass-deformed ABJM theory with a small mass parameter. We also comment on the issue of the relative entropy and the Fisher information in our setup.

KEYWORDS: Gauge-gravity correspondence, M-Theory, Supersymmetric Gauge Theory

ARXiv EPRINT: 1712.09101 


\section{Contents}

1 Introduction 1

2 Construction of 4-dimensional gravity theory 3

2.1 Field equations at quadratic order 4

$\begin{array}{ll}2.2 & \text { Expansion in spherical harmonics }\end{array}$

2.3 The 4-dimensional graviton equation at $\mu_{0}^{2}$ order 8

2.4 The KK mapping at quadratic order 11

3 HEE from vev and source 14

$\begin{array}{ll}3.1 \text { Vacua of mABJM theory and LLM geometries } & 14\end{array}$

$\begin{array}{lll}3.2 & \text { KK reduction of the LLM geometries } & 16\end{array}$

$\begin{array}{lll}3.3 & \text { One-point function for the CPO with } \Delta=1 & 17\end{array}$

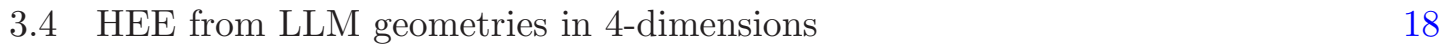

$\begin{array}{lll}\text { 3.4.1 HEE from pure AdS geometry in 4-dimensions } & 19\end{array}$

3.5 Variation of HEE from LLM geometries in 4-dimensions 20

3.6 HEE from holographic mapping of vev and source 21

3.7 Comments on relative entropy and Fisher information 23

4 Gravity from entanglement and RG flow $\quad 24$

4.1 Emergent gravity from relevant perturbations in CFT 24

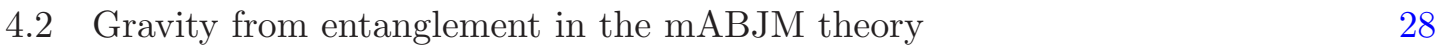

$\begin{array}{lll}5 & \text { Conclusion } & 30\end{array}$

\section{Introduction}

The holographic approach has become a very efficient technique in computing the entanglement entropy (EE) which is rather challenging in a direct path integral approach in quantum field theories (QFTs) in more than two dimensions. The holographic calculation of the EE, which was proposed by Ryu and Takayanagi (RT) [1,2] and its covariant generalization [3], drew much attention due to its elegance and implications in condensed matter physics and gravity theories.

More recently, the idea of the holographic entanglement entropy (HEE) caught more attention because of its importance in unlocking some mysteries of the gauge/gravity correspondence [4-6] itself. The RT formula relates the EE $\left(S_{A}\right)$ of a $(d-1)$-dimensional spatial subregion $A$ in the vacuum state of a $d$-dimensional conformal field theory (CFT), which is living on the boundary of $(d+1)$-dimensional AdS space, to a surface with minimum area in the bulk of the AdS space with the same boundary as the subregion $A$. This creates a 
connection between the EE of QFT and the emergent spacetime geometry of the dual gravity theory. The topic of the emergence of gravity from quantum entanglement has shown some significant progresses in recent years $[1,2,7-9]$. Much of the progress in this direction esteemed from the first law of $\mathrm{EE}\left(\delta S_{A}=\delta\left\langle H_{A}\right\rangle\right)$ [10-13], which equates the variation of $\mathrm{EE}$ due to a transition from a vacuum state of a CFT to some nearby state, and the variation of the vacuum expectation value (vev) of some characteristic Hamiltonian associated with the subregion $A$, which is known as the modular Hamiltonian. For a ball-shaped subregion $A$ on the boundary of an asymptotically AdS space, it was shown that the first law of EE is equivalent to a linearized Einstein equation on the AdS background [14-16]. Alternative approaches and extenstions of this phenomenon were given in [17-20]. See also [21] for the emergence of nonlinear gravitational equations from a broad class of CFTs via the EE.

In this paper, we pursue this phenomena of the emergent spacetime geometry in the context of the 3-dimensional mass-deformed Aharony-Bergman-Jefferis-Meldacena (mABJM) theory $[22,23]$ and the dual gravity theory. The mABJM theory is obtained from the original $\mathcal{N}=6$ ABJM theory [24] in 3-dimensions by a relevant deformation which preserves the full supersymmetry. The dual gravity theory is the 11-dimensional supergravity on the Lin-Lunin-Maldacena (LLM) geometry [25] with $\mathbb{Z}_{k}$ orbifold and $\mathrm{SO}(2,1) \times \mathrm{SO}(4) / \mathbb{Z}_{k} \times \mathrm{SO}(4) / \mathbb{Z}_{k}$ isometry, which is asymptotically $\mathrm{AdS}_{4} \times S^{7} / \mathbb{Z}_{k}$. In $[26,27]$, we have presented a compelling evidence for the gauge/gravity duality between the mABJM theory and the 11-dimensional gravity on the LLM geometry. This was achieved by applying the gauge/gravity dictionary $[5,6]$ to the vevs of the chiral primary operator (CPO) with conformal dimension $\Delta=1$ for all supersymmetric vacua of the mABJM theory and the 4-dimensional dual scalar modes obtained from the procedure of the Kaluza-Klein (KK) holography [28-30] of the 11-dimensional supergravity. We found an exact agreement between the results from the both sides in the large $N$ limit. ${ }^{1}$

In order to show the exact dual relation in $[26,27]$, we mainly dealt with the matter fields, such as scalar, vector, and tensor fields, without considering the 4-dimensional metric. In this paper, however, we construct the 4-dimensional Einstein-Hilbert action with matter from the KK reduction of the 11-dimensional gravity on the LLM geometries with $\mathbb{Z}_{k}$ orbifold. The matter content of the 4-dimensional theory is determined by the asymptotic behaviour of the LLM solutions. At quadratic order in the mass parameter $\mu_{0}$ of the LLM solutions, the matter sector is composed of only one scalar field $(\Psi)$ and one pseudoscalar field $(T)$. These matter fields as well as the 4-dimensional graviton field $\left(H_{\mu \nu}\right)$ are related to the 11-dimensional KK modes by some non-trivial field redefinitions, often called the KK maps. Solution of the linearized Einstein equation for $\left(H_{\mu \nu}\right)$ with the energy-momentum tensor for the two scalar fields is asymptotically $\mathrm{AdS}_{4}$ and encodes the information of all LLM geometries in the small mass limit.

The presence of the two matter fields in the 4-dimensional gravity theory corresponds to the deformation of the ABJM theory by two relevant operators, which are a CPO of conformal dimension one $\left(\mathcal{O}^{(1)}\right)$ dual to $\Psi$ and a gauge invariant operator of conformal dimension two $\left(\tilde{\mathcal{O}}^{(2)}\right)$ dual to $T$. In this setting, where the CFT with relevant deforma-

\footnotetext{
${ }^{1}$ See also [31] for the extension to the vevs of the CPO with conformal dimension $\Delta=2$.
} 
tion and its dual gravity theory are explicitly known, we investigate the emergent gravity phenomena away from the UV fixed point by computing the variation of $\mathrm{EE}\left(\delta S_{A}\right)$ of the disk-shaped region $A$ in terms of the RT formula $[1,2]$ in the gravity theory and the path integral method developed in [32] in QFT side.

The EE for a CFT with relevant deformation is calculated using the path integral method [32-35], in which the EE is represented in terms of correlation functions. See also [36-39]. In particular, in [32] the author showed that the EE of the $d$-dimensional CFT with a relevant deformation can be regarded as a conserved charge in an emergent (auxiliary) $(d+1)$-dimensional gravity theory. The same quantity was also computed in the dual gravity theory [40-45] using the RT formula. The EE from the direct path integral approach is exactly the same as the HEE in RT formula with the metric satisfying the linearized Einstein equation in the presence of a nonvanishing energy-momentum tensor.

In our case, the deformations by the two relevant operators, $\mathcal{O}^{(1)}$ and $\tilde{\mathcal{O}}^{(2)}$, belong to the $\Delta<d / 2$ and $\Delta>d / 2$ cases, which require separate treatments [32, 37, 40]. In this setup, we calculate the variation of the $\mathrm{EE}\left(\delta S_{A}\right)$ of the disk region $A$ up to quadratic order in the deformation parameter in the QFT side and the gravity side separately. In the QFT side, we rely on the exact dual relation established in $[26,27]$ and use the method developed in [32], while in the gravity side we use the RT formula for the 4-dimensional metric in the presence of the matter fields. ${ }^{2}$ We show that the $\delta S_{A}$ 's obtained from both sides are equal only when the Einstein equation in the 4-dimensional gravity theory is satisfied. It implies that the asymptotic limit of the LLM geometry is actually the emergent gravity in the small mass expansion.

The remaining part of the paper is organized as follows. In section 2, we construct the 4-dimensional gravity from the KK reduction of the 11-dimensional supergravity. We also setup the non-trivial KK maps between the 11-dimensional fields and the 4-dimensional fields. In section 3, we use the RT formula to calculate the HEE from an asymptotically $\mathrm{AdS}_{4}$ metric, which is obtained from the KK reduction of the LLM solution. We then establish the relation between the variation of the HEE and the vev of a conformal dimension one CPO plus the source of a conformal dimension two gauge invariant operator in mABJM theory. In section 4, we review the path integral methods necessary to obtain the EE in a CFT with some relevant deformation. We apply these methods to our setup and obtain the variation of the EE for mABJM theory at quadratic order in the deformation parameter. We use the results in the first law of EE at quadratic order, to show the emergence of the gravity in 4-dimensions in a top-down approach from the quantum entanglement of the 3-dimensional mABJM theory. In section 5, we draw some conclusions and discuss some future directions.

\section{Construction of 4-dimensional gravity theory}

In this section, we construct a 4-dimensional gravity theory on $\mathrm{AdS}_{4}$ background from the KK reduction of the 11-dimensional supergravity theory. In particular, we focus on a 4-

\footnotetext{
${ }^{2}$ The HEE obtained from the 4-dimensional metric agrees with the HEE which was obtained from the 11-dimensional LLM geometries before the KK reduction [46]. See also [47, 48].
} 
dimensional gravity theory whose matter content is determined by the LLM solution with $\mathrm{SO}(2,1) \times \mathrm{SO}(4) / \mathbb{Z}_{k} \times \mathrm{SO}(4) / \mathbb{Z}_{k}$ isometry. In $[26,27]$ we performed the $\mathrm{KK}$ reduction at linear order and obtained the linearized KK maps among gauge invariant 11-dimensional and 4-dimensional fields. The linearized reduction involves the truncation of the field equations at linear order in the fluctuations on the $\mathrm{AdS}_{4} \times \mathrm{S}^{7} / \mathbb{Z}_{k}$ solution of the 11-dimensional supergravity. The linearized field equations are solved by the asymptotic expansion of the LLM solutions at linear order in the mass parameter $\mu_{0}$. However, to solve the field equations at $\mu_{0}^{2}$ order or higher, the linearized KK reduction is not sufficient. In this section, we construct the nonlinear KK mapping up to $\mu_{0}^{2}$ order by truncating the field equations at quadratic order in the fluctuations.

\subsection{Field equations at quadratic order}

The functional variation of the bosonic part of the 11-dimensional supergravity action gives the following equations for the metric and the 3 -form gauge field,

$$
\begin{aligned}
& \mathbf{R}_{p q}-\frac{1}{2} \mathbf{g}_{p q} \mathbf{R}=\frac{1}{48}\left(-\frac{1}{2} \mathbf{g}_{p q} \mathbf{F}_{r s t u} \mathbf{F}^{r s t u}+4 \mathbf{F}_{p s t u} \mathbf{F}_{q}^{s t u}\right), \\
& \partial_{p}\left(\sqrt{-\mathbf{g}} \mathbf{F}^{p q r s}\right)+\frac{1}{2 \cdot(4 !)^{2}} \tilde{\epsilon}^{p_{1} \cdots p_{4} q_{1} \cdots q_{4} q r s} \mathbf{F}_{p_{1} \cdots p_{4}} \mathbf{F}_{q_{1} \cdots q_{4}}=0,
\end{aligned}
$$

where we used the index notation $(p, q, r, \cdots=0,1, \cdots, 10)$ and $\tilde{\epsilon}^{012 \cdots 10}=-1$ is the LeviCivita symbol. ${ }^{3}$ The $\mathrm{AdS}_{4} \times S^{7}$ solution of (2.1) is given by ${ }^{4}$

$$
\begin{aligned}
d s^{2} & =\frac{L^{2}}{4 z^{2}}\left(-d t^{2}+d w_{1}^{2}+d w_{2}^{2}+d z^{2}\right)+L^{2} d s_{S^{7}}^{2}, \\
F_{\mu \nu \rho \sigma} & =-\frac{6}{L} \epsilon_{\mu \nu \rho \sigma}, \text { and it is zero otherwise, }
\end{aligned}
$$

where we split the 11-dimensional indices into the $\mathrm{AdS}_{4}$ indices $(\mu, \nu, \cdots=0, \cdots, 3)$ and the $S^{7}$ indices $(a, b, \cdots=4, \cdots, 10)$. Here $\epsilon_{\mu \nu \rho \sigma}=\sqrt{\left|g_{\mathrm{AdS}_{4} \mid}\right|} \tilde{\epsilon}_{\mu \nu \rho \sigma}$ is the Levi-Civita tensor for the $\mathrm{AdS}_{4}$ space and $L$ is the radius of $S^{7}$.

To obtain nonlinear field equations from (2.1), we perturb the fields around $\mathrm{AdS}_{4} \times S^{7}$ background by expressing the 11-dimensional metric and the 4-form field strength as

$$
\mathbf{g}_{p q}=g_{p q}+h_{p q}, \quad \mathbf{F}_{p q r s}=F_{p q r s}+f_{p q r s},
$$

and keep all terms up to quadratic order in the fluctuations $h_{p q}$ and $f_{p q r s}$. Applying such perturbation to the metric equation in (2.1), we obtain

$$
\begin{aligned}
\nabla^{r} & \nabla_{p} h_{q r}+\nabla^{r} \nabla_{q} h_{p r}-\nabla^{2} h_{p q}-\nabla_{q} \nabla_{p} h_{r}^{r}-R h_{p q}-g_{p q}\left(-R^{r s} h_{r s}+\nabla^{r} \nabla^{s} h_{r s}-\nabla^{2} h_{r}^{r}\right) \\
& +\frac{1}{48}\left(F_{r s t u} F^{r s t u} h_{p q}-4 g_{p q} h_{r s} F_{t u v}^{r} F^{s t u v}\right)+\frac{1}{24} g_{p q} f_{p q r s} F^{p q r s}-\frac{1}{2} h_{r s} F_{p t u}^{r} F_{q}{ }^{s t u} \\
& -\frac{1}{6}\left(f_{p r s t} F_{q}{ }^{r s t}+F_{p r s t} f_{q}^{r s t}\right)+Q_{p q}=0
\end{aligned}
$$

\footnotetext{
${ }^{3}$ We use a notation in which the quantities in 11-dimensional supergravity are denoted by bold font symbols whereas the normal font symbols are reserved for $\mathrm{AdS}_{4} \times S^{7}$ values of those quantities.

${ }^{4}$ For the $\mathbb{Z}_{k}$ orbifold, we discuss in the subsection 2.2 .
} 
where the indices are raised (lowered) by the $\mathrm{AdS}_{4} \times S^{7}$ metric, and the covariant derivatives are also those of the background. Here $Q_{p q}$ denotes terms which are quadratic in the fluctuations,

$$
\begin{aligned}
& Q_{p q}=-\nabla_{r}\left(h^{r s}\left[\nabla_{p} h_{s q}+\nabla_{q} h_{s p}-\nabla_{s} h_{p q}\right]\right)+\frac{1}{2} \nabla_{q} h^{r s} \nabla_{p} h_{r s}+h^{r s} \nabla_{q} \nabla_{p} h_{r s} \\
& +\frac{1}{2} \nabla^{r} h_{s}^{s}\left[\nabla_{p} h_{r q}+\nabla_{q} h_{r p}-\nabla_{r} h_{p q}\right]+\nabla^{r} h^{s} \nabla_{r} h_{s p}-\nabla^{r} h_{q}^{s} \nabla_{s} h_{p r}-g_{p q} R_{r s} h^{r t} h^{s}{ }_{t} \\
& +\frac{1}{2} g_{p q} \nabla_{r}\left(h^{r s}\left[2 \nabla^{t} h_{s t}-\nabla_{s} h_{t}^{t}\right]\right)-\frac{3}{4} g_{p q} \nabla^{t} h^{r s} \nabla_{t} h_{r s}+\frac{1}{2} g_{p q} \nabla^{r} h^{s t} \nabla_{s} h_{t r}-\frac{1}{2} g_{p q} h^{r s} \nabla^{2} h_{r s} \\
& -\frac{1}{4} g_{p q} \nabla^{r} h_{s}^{s}\left[2 \nabla^{s} h_{r s}-\nabla_{r} h_{t}^{t}\right]+\frac{1}{2} g_{p q} h^{r s}\left(\nabla^{t} \nabla_{r} h_{t s}+\nabla^{t} \nabla_{s} h_{t r}-\nabla^{2} h_{r s}-\nabla_{r} \nabla_{s} h_{t}^{t}\right) \\
& +h_{p q} h^{r s} R_{r s}-h_{p q}\left(\nabla^{r} \nabla^{s} h_{r s}-\nabla^{2} h_{r}^{r}\right) \\
& +\frac{1}{12}\left(g_{p q} F_{r s t u} F^{r s t}{ }_{s^{\prime}} h^{u v} h_{v}^{s^{\prime}}+\frac{3}{2} g_{p q} F_{r s t u} F^{r s}{ }_{r^{\prime} s^{\prime}} h^{t r^{\prime}} h^{u s^{\prime}}-g_{p q} F_{r s t u} f^{r s t}{ }_{s^{\prime}} h^{u s^{\prime}}\right. \\
& \left.\quad+\frac{1}{2} h_{p q} f_{r s t u} F^{r s t u}-h_{p q} F_{r s t u} F^{r s t}{ }_{s^{\prime}} h^{u s^{\prime}}+\frac{1}{4} g_{p q} f_{r s t u} f^{r s t u}-g_{p q} f_{r s t u} F^{r s t}{ }_{s^{\prime}} h^{u s^{\prime}}\right) \\
& -\frac{1}{2}\left(F_{p s t u} F_{q}{ }^{s t}{ }_{s^{\prime}} h^{u v} h_{v}^{s^{\prime}}+F_{p s t u} F_{q}{ }^{s} r_{s^{\prime}} h^{t r^{\prime}} h^{u s^{\prime}}-F_{p s t u} f_{q}{ }^{s t}{ }_{s^{\prime}} h^{u s^{\prime}}-f_{p s t u} F_{q}{ }^{s t}{ }_{s^{\prime}} h^{u s^{\prime}}+\frac{1}{3} f_{p s t u} f_{q}{ }^{s t u}\right) .
\end{aligned}
$$

Similarly, from the gauge field equation in (2.1) we obtain

$$
\nabla_{p}\left(h^{t}{ }_{t} F^{p q r s}\right)+2 \nabla_{p}\left(4 F_{s^{\prime}}^{[p q r} h^{s] s^{\prime}}+f^{p q r s}\right)+\frac{2}{\sqrt{-g}} \frac{1}{(4 !)^{2}} \tilde{\epsilon}^{p_{1} \cdots p_{4} q_{1} \cdots q_{4} q r s} f_{p_{1} \cdots p_{4}} F_{q_{1} \cdots q_{4}}+P^{q r s}=0,
$$

where

$$
\begin{aligned}
P^{q r s}= & -\frac{1}{2} \nabla_{p}\left[\left(h_{t u} h^{t u}-\frac{1}{2}\left(h^{t}{ }_{t}\right)^{2}\right) F^{p q r s}\right]-8 \nabla_{p}\left[F_{s^{\prime}}{ }^{[p q r} h^{s] t} h_{t}^{s^{\prime}}-\frac{3}{2} F^{r^{\prime} s^{\prime}[p q} h_{r^{\prime}}^{r} h_{s^{\prime}}-f_{s^{\prime}}{ }^{[p q r} h^{s] s^{\prime}}\right] \\
& +\nabla_{p}\left[h^{t}{ }_{t}\left(4 F_{s^{\prime}}{ }^{[p q r} h^{s] s^{\prime}}+f^{p q r s}\right)\right]+\frac{1}{\sqrt{-g}} \frac{1}{(4 !)^{2}} \tilde{\epsilon}^{p_{1} \cdots p_{4} q_{1} \cdots q_{4} q r s} f_{p_{1} \cdots p_{4}} f_{q_{1} \cdots q_{4}} .
\end{aligned}
$$

For later convenience we write (2.4) and (2.6) by separating the $\mathrm{AdS}_{4}$ and the $S^{7}$ indices and also inserting the $\mathrm{AdS}_{4} \times S^{7}$ background information given in (2.2). One can write those quadratic equations in generic gauge, however, having the LLM solutions in mind, we simplify the equations by setting $h_{\mu a}$ and $f_{\mu \nu a b}$ to zero. Then we obtain

$$
\begin{aligned}
& \nabla^{\rho} \nabla_{\mu} h_{\nu \rho}+\nabla^{\rho} \nabla_{\nu} h_{\mu \rho}-\left(\nabla^{\rho} \nabla_{\rho}+\nabla^{a} \nabla_{a}\right) h_{\mu \nu}-\nabla_{\mu} \nabla_{\nu}\left(h_{\rho}^{\rho}+h_{a}^{a}\right)+\frac{24}{L^{2}} h_{\mu \nu} \\
& -g_{\mu \nu}\left[\frac{30}{L^{2}} h_{\rho}^{\rho}-\frac{6}{L^{2}} h_{a}^{a}+\nabla^{\rho} \nabla^{\sigma} h_{\rho \sigma}+\nabla^{a} \nabla^{b} h_{a b}-\left(\nabla^{\rho} \nabla_{\rho}+\nabla^{a} \nabla_{a}\right)\left(h_{\sigma}^{\sigma}+h_{b}^{b}\right)\right] \\
& \quad-\frac{1}{4 L} g_{\mu \nu} f_{\rho \sigma \tau \lambda} \epsilon^{\rho \sigma \tau \lambda}+\frac{1}{L}\left(f_{\mu \rho \sigma \tau} \epsilon_{\nu}^{\rho \sigma \tau}+f_{\nu \rho \sigma \tau} \epsilon_{\mu}^{\rho \sigma \tau}\right)+Q_{\mu \nu}=0 \\
& \nabla^{\rho} \nabla_{a} h_{\mu \rho}+\nabla^{b} \nabla_{\mu} h_{a b}-\nabla_{\mu} \nabla_{a}\left(h^{\rho}{ }_{\rho}+h_{b}^{b}\right)+\frac{1}{L} f_{a \rho \sigma \tau} \epsilon_{\mu}^{\rho \sigma \tau}+Q_{\mu a}=0 \\
& \nabla^{c} \nabla_{a} h_{b c}+\nabla^{c} \nabla_{b} h_{a c}-\left(\nabla^{\rho} \nabla_{\rho}+\nabla^{c} \nabla_{c}\right) h_{a b}-\nabla_{a} \nabla_{b}\left(h^{\rho}{ }_{\rho}+h_{c}^{c}\right)-\frac{12}{L^{2}} h_{a b}-\frac{1}{4 L} g_{a b} f_{\rho \sigma \tau \lambda} \epsilon^{\rho \sigma \tau \lambda} \\
& \quad-g_{a b}\left(-\frac{6}{L^{2}} h_{\rho}^{\rho}-\frac{6}{L^{2}} h_{c}^{c}+\nabla^{\rho} \nabla^{\sigma} h_{\rho \sigma}+\nabla^{c} \nabla^{d} h_{c d}-\left(\nabla^{\rho} \nabla_{\rho}+\nabla^{c} \nabla_{c}\right)\left(h_{\sigma}^{\sigma}+h_{d}^{d}\right)\right)+Q_{a b}=0
\end{aligned}
$$


and

$$
\begin{aligned}
& \nabla_{\sigma} f^{\sigma \mu \nu \rho}+\nabla_{a} f^{a \mu \nu \rho}-\frac{3}{L}\left(\nabla_{\sigma} h_{\lambda}^{\lambda}\right) \epsilon^{\sigma \mu \nu \rho}-\frac{3}{L}\left(\nabla_{\sigma} h_{a}^{a}\right) F^{\sigma \mu \nu \rho}-\frac{24}{L} \nabla_{\lambda}\left(h_{\sigma}^{[\lambda} \epsilon^{\mu \nu \rho] \sigma}\right)+P^{\mu \nu \rho}=0, \\
& \nabla_{\sigma} f^{\sigma \mu \nu a}+P^{\mu \nu a}=0, \quad \nabla_{c} f^{c \mu a b}+P^{\mu a b}=0, \\
& \nabla_{\sigma} f^{\sigma a b c}+\nabla_{d} f^{d a b c}+\frac{1}{(4 !)^{2}} \epsilon^{a_{1} \cdots a_{4} \nu_{1} \cdots \nu_{4} a b c} f_{a_{1} \cdots a_{4}} F_{\nu_{1} \cdots \nu_{4}}+P^{a b c}=0 .
\end{aligned}
$$

\subsection{Expansion in spherical harmonics}

The KK reduction involves the expansions of the fluctuations $h_{p q}$ and $f_{p q r s}$ in terms of the spherical harmonics on $S^{7}$. Here we are interested in the asymptotic limit of the LLM geometry with $\mathrm{SO}(2,1) \times \mathrm{SO}(4) / \mathbb{Z}_{k} \times \mathrm{SO}(4) / \mathbb{Z}_{k}$ isometry. In that case, we need to consider expansion in terms of the spherical harmonics on $S^{7} / \mathbb{Z}_{k}$ with $\mathrm{SO}(4) / \mathbb{Z}_{k} \times \mathrm{SO}(4) / \mathbb{Z}_{k}$ symmetry. In the presence of such symmetry, the metric on the $S^{7} / \mathbb{Z}_{k}$ is written as

$$
d s_{S^{7} / \mathbb{Z}_{k}}^{2}=d \tau^{2}+\frac{d \theta^{2}+\sin ^{2} \theta d \phi^{2}+(d \psi+\cos \theta d \phi)^{2}}{4}+\frac{d \tilde{\theta}^{2}+\sin ^{2} \tilde{\theta} d \tilde{\phi}^{2}+(d \tilde{\psi}+\cos \tilde{\theta} d \tilde{\phi})^{2}}{4},
$$

with ranges of the angles, $0 \leq \theta, \tilde{\theta} \leq \pi, 0 \leq \phi, \tilde{\phi} \leq 2 \pi, 0 \leq \psi, \tilde{\psi} \leq \frac{4 \pi}{k}$. The $\mathbb{Z}_{k}$ orbifolding acts as $(\psi, \tilde{\psi}) \rightarrow\left(\psi+\frac{4 \pi}{k}, \tilde{\psi}+\frac{4 \pi}{k}\right)[49,50]$. The spherical harmonics with the $\mathrm{SO}(4) / \mathbb{Z}_{k} \times$ $\mathrm{SO}(4) / \mathbb{Z}_{k}$ symmetry are dependent only on the $\tau$ coordinate, and they are the same with and without the orbifolding. This implies that expansions of the fluctuations $h_{p q}$ and $f_{p q r s}$ in terms of these spherical harmonics are the same with and without the orbifolding.

In $[26,27]$, we have written a complete form of the expansions in terms of the spherical harmonics on $S^{7}$. For clarity of presentation, let us recall the expansion,

$$
\begin{aligned}
h_{\mu \nu}(x, y) & =h_{\mu \nu}^{I_{1}}(x) Y^{I_{1}}(y), \\
h_{\mu a}(x, y) & =v_{\mu}^{I_{7}}(x) Y_{a}^{I_{7}}(y)+s_{\mu}^{I_{1}}(x) \nabla_{a} Y^{I_{1}}(y), \\
h_{(a b)}(x, y) & =t^{I_{27}}(x) Y_{(a b)}^{I_{27}}(y)+v^{I_{7}}(x) \nabla_{(a} Y_{b)}^{I_{7}}(y)+s^{I_{1}}(x) \nabla_{(a} \nabla_{b)} Y^{I_{1}}(y), \\
h_{a}^{a}(x, y) & =\phi^{I_{1}}(x) Y^{I_{1}}(y) \\
f_{\mu \nu \rho \sigma}(x, y) & =4 \nabla_{[\mu} s_{\nu \rho \sigma]}^{I_{1}}(x) Y^{I_{1}}(y), \\
f_{\mu \nu \rho a}(x, y) & =3 \nabla_{[\mu} v_{\nu \rho]}^{I_{7}}(x) Y_{a}^{I_{7}}(y)-s_{\mu \nu \rho}^{I_{1}}(x) \nabla_{a} Y^{I_{1}}(y), \\
f_{\mu \nu a b}(x, y) & =2 \nabla_{[\mu} t_{\nu]}^{I_{21}}(x) Y_{[a b]}^{I_{21}}(y)+2 v_{\mu \nu}^{I_{7}}(x) \nabla_{[a} Y_{b]}^{I_{7}}(y), \\
f_{\mu a b c}(x, y) & =\nabla_{\mu} t^{I_{35}}(x) Y_{[a b c]}^{I_{35}}(y)-3 t_{\mu}^{I_{21}}(x) \nabla_{[a} Y_{b c]}^{I_{21}}(y), \\
f_{a b c d}(x, y) & =4 t^{I_{35}}(x) \nabla_{[a} Y_{b c d]}^{I_{35}}(y),
\end{aligned}
$$

where $x$ denotes the $\mathrm{AdS}_{4}$ coordinates and $y$ denotes the $S^{7}$ coordinates. For the details about the spherical harmonics on $S^{7}$, see $[26,27]$. The parenthesis of two indices $(a b)$ means symmetrized traceless combination, while the square bracket $[a b \cdots]$ denotes complete antisymmetrization of indices. Eventually, we will identify the fluctuations $h_{p q}$ and $f_{\text {pqrs }}$ with the deviations of the LLM geometries from the $\mathrm{AdS}_{4} \times S^{7}$ background. In the gauge choice of the LLM solutions, $h_{\mu a}$ and $f_{\mu \nu a b}$ are zero and as a result most of the KK towers in (2.12) are absent. Therefore, we use a truncated expansion,

$$
\begin{aligned}
h_{\mu \nu}(x, y) & =h_{\mu \nu}^{I_{1}}(x) Y^{I_{1}}(y), & h^{\rho}{ }_{\rho}(x, y) & =h^{I_{1}}(x) Y^{I_{1}}(y), \\
h_{a}^{a}(x, y) & =\phi^{I_{1}}(x) Y^{I_{1}}(y), & h_{(a b)} & =s^{I_{1}}(x) \nabla_{(a} \nabla_{b)} Y^{I_{1}}(y),
\end{aligned}
$$


and

$$
\begin{aligned}
& f_{\mu \nu \rho \sigma}(x, y)=4 \nabla_{[\mu} s_{\nu \rho \sigma]}^{I_{1}}(x) Y^{I_{1}}(y), \quad f_{\mu \nu \rho a}(x, y)=-s_{\mu \nu \rho}^{I_{1}}(x) \nabla_{a} Y^{I_{1}}(y), \\
& f_{\mu a b c}(x, y)=\nabla_{\mu} t^{I_{35}}(x) Y_{a b c}^{I_{35}}(y), \quad f_{a b c d}(x, y)=4 t^{I_{35}}(x) \nabla_{[a} Y_{b c d]}^{I_{35}}(y) .
\end{aligned}
$$

This truncation is not dictated by some symmetry, which would have been a requirement in order to have a consistent truncation if one follows the line of thought of ref. [51]. However, the equations obtained from the truncated expansion are consistent at quadratic order in the fluctuations. The reason is that those equations are solved order by order in the mass parameter $\left(\mu_{0}\right)$ of the LLM solutions, and the modes which are omitted from the expansion are all vanishing, at least up to $\mu_{0}^{2}$-order [26, 27].

Plugging (2.13) and (2.14) into (2.8) and then projecting on the scalar harmonics $Y^{I_{1}}$, we obtain

$$
\begin{aligned}
& -\left(\square+\Lambda^{I_{1}}-\frac{24}{L^{2}}\right) h_{\mu \nu}^{I_{1}}+\nabla^{\rho} \nabla_{\mu} h_{\nu \rho}^{I_{1}}+\nabla^{\rho} \nabla_{\nu} h_{\mu \rho}^{I_{1}}-\nabla_{\mu} \nabla_{\nu}\left(h^{I_{1}}+\phi^{I_{1}}\right) \\
& \quad+g_{\mu \nu}\left(\square+\Lambda^{I_{1}}-\frac{30}{L^{2}}\right) h^{I_{1}}+g_{\mu \nu}\left(\square+\frac{6}{7} \Lambda^{I_{1}}+\frac{6}{L^{2}}\right) \phi^{I_{1}}-g_{\mu \nu}\left(\frac{6}{7} \Lambda^{I_{1}}+\frac{6}{L^{2}}\right) \Lambda^{I_{1}} s^{I_{1}} \\
& -g_{\mu \nu} \nabla^{\rho} \nabla^{\sigma} h_{\rho \sigma}^{I_{1}}+\frac{1}{L} g_{\mu \nu} \nabla^{\rho} t_{\rho}^{I_{1}}+Q_{\mu \nu}^{I_{1}}=0,
\end{aligned}
$$

where $\square \equiv \nabla_{\mu} \nabla^{\mu}, Q_{\mu \nu}^{I_{1}}=\frac{1}{\omega_{7}} \int_{S^{7}} Q_{\mu \nu} Y^{I_{1}}$ with the unit volume of the $S^{7}, \omega_{7}$, and we have set $s_{\mu \nu \rho}^{I_{1}}=\frac{1}{3 !} \epsilon_{\mu \nu \rho}{ }^{\sigma} t_{\sigma}^{I_{1}}$, and $\Lambda^{I_{1}}=-\frac{I_{1}\left(I_{1}+6\right)}{L^{2}}$ is the eigenvalue corresponding to the scalar harmonics $Y^{I_{1}}$. Taking the trace of the above equation, we obtain

$$
\begin{aligned}
& \left(2 \square+3 \Lambda^{I_{1}}-\frac{96}{L^{2}}\right) h^{I_{1}}-2 \nabla^{\mu} \nabla^{\nu} h_{\mu \nu}^{I_{1}}+3\left(\square+\frac{8}{7} \Lambda^{I_{1}}+\frac{8}{L^{2}}\right) \phi^{I_{1}}+\frac{4}{L} \nabla^{\rho} t_{\rho}^{I_{1}} \\
& -24 \Lambda^{I_{1}}\left(\frac{1}{7} \Lambda^{I_{1}}+\frac{1}{L^{2}}\right) s^{I_{1}}+Q^{I_{1}}=0
\end{aligned}
$$

with $Q^{I_{1}}=g^{\mu \nu} Q_{\mu \nu}^{I_{1}}$. From (2.9), we obtain the following equation by projecting on $\nabla^{a} Y^{I_{1}}$ with $I_{1} \neq 0$,

$$
-\left(\frac{6}{7} \Lambda^{I_{1}}+\frac{6}{L^{2}}\right) \nabla_{\mu} s^{I_{1}}+\frac{6}{7} \nabla_{\mu} \phi^{I_{1}}-\nabla^{\nu} h_{\mu \nu}^{I_{1}}+\nabla_{\mu} h^{I_{1}}-\frac{1}{L} t_{\mu}^{I_{1}}+Q_{\mu}^{I_{1}}=0,
$$

where $Q_{\mu}^{I_{1}}=\frac{1}{\omega_{7}} \int_{S^{7}} Q_{\mu a} \nabla^{a} Y^{I_{1}}$. From (2.10), we obtain two scalar equations by projecting on $g^{a b} Y^{I_{1}}$ and $\nabla^{(a} \nabla^{b)} Y^{I_{1}}$, in the latter case $I_{1} \neq 0$,

$$
\begin{aligned}
& \frac{6}{7}\left(\square+\frac{5}{7} \Lambda^{I_{1}}+\frac{5}{L^{2}}\right) \phi^{I_{1}}+\left(\square+\frac{6}{7} \Lambda^{I_{1}}+\frac{6}{L^{2}}\right) h^{I_{1}}-\nabla^{\mu} \nabla^{\nu} h_{\mu \nu}^{I_{1}}-\frac{1}{L} \nabla^{\rho} t_{\rho}^{I_{1}} \\
& \quad-\frac{30}{7} \Lambda^{I_{1}}\left(\frac{\Lambda^{I_{1}}}{7}+\frac{1}{L^{2}}\right) s^{I_{1}}+\tilde{Q}^{I_{1}}=0, \\
& 6 \Lambda^{I_{1}}\left(\frac{\Lambda^{I_{1}}}{7}+\frac{1}{L^{2}}\right)\left[\left(\square-\frac{5}{7} \Lambda^{I_{1}}\right) s^{I_{1}}+h^{I_{1}}+\frac{5}{7} \phi^{I_{1}}\right]-\hat{Q}^{I_{1}}=0,
\end{aligned}
$$

where $\tilde{Q}^{I_{1}}=\frac{1}{\omega_{7}} \int_{S^{7}} Q_{a b} g^{a b} Y^{I_{1}}$ and $\hat{Q}^{I_{1}}=\frac{1}{\omega_{7}} \int_{S^{7}} Q_{a b} \nabla^{(a} \nabla^{b)} Y^{I_{1}}$. 
Similarly, inserting (2.13) and (2.14) into (2.11), and projecting on the appropriate spherical harmonic elements, we obtain the following set of equations

$$
\begin{aligned}
& 4 \nabla^{\sigma} \nabla_{[\sigma} s_{\mu \nu \rho]}^{I_{1}}+\Lambda^{I_{1}} s_{\mu \nu \rho}^{I_{1}}-\frac{3}{L} \epsilon_{\sigma \mu \nu \rho} \nabla^{\sigma}\left(h^{I_{1}}+\phi^{I_{1}}\right)-\frac{24}{L} \nabla^{\sigma} h_{\lambda[\sigma}^{I_{1}} \epsilon_{\mu \nu \rho]}{ }^{\lambda}+P_{\mu \nu \rho}^{I_{1}}=0, \\
& \Lambda^{I_{1}} \nabla^{\rho} s_{\rho \mu \nu}^{I_{1}}+P_{\mu \nu}^{I_{1}}=0, \quad\left(I_{1} \neq 0\right), \\
& \left(\square-\frac{12}{L^{2}}+\Lambda^{I_{35}} \pm \frac{6\left(I_{35}+3\right)}{L^{2}}\right) t_{ \pm}^{I_{35}}+P^{I_{35}}=0,
\end{aligned}
$$

where $\Lambda^{I_{35}}=-\frac{I_{35}\left(I_{35}+6\right)-3}{L^{2}}$ and

$$
P_{\mu \nu \rho}^{I_{1}}=\frac{1}{\omega_{7}} \int_{S^{7}} P_{\mu \nu \rho} Y^{I_{1}}, \quad P_{\mu \nu}^{I_{1}}=\frac{1}{\omega_{7}} \int_{S^{7}} P_{\mu \nu a} \nabla^{a} Y^{I_{1}}, \quad P^{I_{35}}=\frac{1}{\omega_{7}} \int_{S^{7}} P^{a b c} Y_{a b c}^{I_{35}} .
$$

Here we have used the relation, $\epsilon_{a b c}{ }^{a_{1} a_{2} a_{3} a_{4}} \nabla_{a_{1}} Y_{a_{2} a_{3} a_{4}}^{I_{35}}= \pm 3 ! \frac{\left(I_{35}+3\right)}{L} Y_{a b c}^{I_{35}}$, to obtain the two equations in (2.22). For later convenience let us again set $s_{\mu \nu \rho}^{I_{1}}=\frac{1}{3 !} \epsilon_{\mu \nu \rho} t_{\lambda}^{I_{1}}$ and then multiply (2.20) by $\epsilon_{\mu^{\prime}}^{\mu \nu \rho} \nabla_{\nu^{\prime}}$. Thus we obtain

$$
\frac{18}{L} \nabla_{\mu} \nabla_{\nu}\left(-h^{I_{1}}+\phi^{I_{1}}\right)+\Lambda^{I_{1}} \nabla_{\nu} t_{\mu}^{I_{1}}+\nabla_{\mu} \nabla_{\nu} \nabla^{\rho} t_{\rho}^{I_{1}}+\tilde{P}_{\mu \nu}^{I_{1}}=0
$$

where $\tilde{P}_{\mu \nu}^{I_{1}}=\epsilon_{\mu}{ }^{\rho \sigma \lambda} \nabla_{\nu} P_{\rho \sigma \lambda}^{I_{1}}$. The trace of the above equation gives

$$
\frac{18}{L} \square\left(-h^{I_{1}}+\phi^{I_{1}}\right)+\left(\square+\Lambda^{I_{1}}\right) \nabla^{\rho} t_{\rho}^{I_{1}}+\tilde{P}^{I_{1}}=0, \quad \tilde{P}^{I_{1}}=g^{\mu \nu} \tilde{P}_{\mu \nu}^{I_{1}} .
$$

\subsection{The 4-dimensional graviton equation at $\mu_{0}^{2}$ order}

From the quadratic equations in the previous subsection, one can obtain the quadratic order equations of motions for various 4-dimensional gauge invariant KK modes. In $[26,27]$ we have obtained the complete 4-dimensional KK spectrum, which in general is composed of three towers of scalar modes, two towers of pseudoscalar modes, two towers of vector modes, one tower of pseudovector mode, and one tower of spin-two mode. We have also found the linear order equations for these modes in generic gauge. In this paper, we have set $h_{\mu a}$ and $f_{\mu \nu a b}$ to zero and as a result some of the KK towers disappear. Furthermore, to construct the equation of motion for 4-dimensional graviton, we focus on the KK zero modes. To that end, we start from the zero modes of the equations (2.15), (2.16), (2.18), (2.24), and (2.25). Let us rearrange those equations by introducing $u^{I_{1}} \equiv L \nabla^{\rho} t_{\rho}^{I_{1}}$ and $\hat{\psi}^{I_{1}} \equiv 18 h^{I_{1}}-u^{I_{1}}$, as follows

$$
\begin{aligned}
\square h_{\mu \nu}^{0}= & \frac{24}{L^{2}} h_{\mu \nu}^{0}+\nabla^{\rho} \nabla_{\mu} h_{\nu \rho}^{0}+\nabla^{\rho} \nabla_{\nu} h_{\mu \rho}^{0}-\nabla_{\mu} \nabla_{\nu}\left(h^{0}+\phi^{0}\right)-g_{\mu \nu} \frac{4}{3 L^{2}} \hat{\psi}^{0} \\
& +Q_{\mu \nu}^{0}-\frac{1}{9} g_{\mu \nu}\left(Q^{0}+7 \tilde{Q}^{0}\right), \\
\square h^{0}= & -\frac{78}{L^{2}} h^{0}+\frac{6}{L^{2}} \phi^{0}+\frac{5}{L^{2}} u^{0}+\nabla^{\rho} \nabla^{\sigma} h_{\rho \sigma}^{0}+\frac{1}{3}\left(2 Q^{0}-7 \tilde{Q}^{0}\right), \\
\square \phi^{0}= & \frac{14}{3 L^{2}} \hat{\psi}^{0}-\frac{12}{L^{2}} \phi^{0}-\frac{7}{9}\left(Q^{0}-2 \tilde{Q}^{0}\right), \\
\square \hat{\psi}^{0}= & \frac{84}{L^{2}} \hat{\psi}^{0}-\frac{216}{L^{2}} \phi^{0}-14\left(Q^{0}-2 \tilde{Q}^{0}\right)+L \tilde{P}^{0}, \\
& -\nabla_{\mu} \nabla_{\nu} \hat{\psi}^{0}+18 \nabla_{\mu} \nabla_{\nu} \phi^{0}+L \tilde{P}_{\mu \nu}^{0}=0 .
\end{aligned}
$$


We notice that, the linear part of (2.26) is not the 4-dimension linearized Einstein equation, which is given by

$$
\left(L_{E}+\frac{12}{L^{2}}\right) h_{\mu \nu}^{0}=0
$$

with $L_{E} h_{\mu \nu}^{0}=\frac{1}{2}\left(-\square h_{\mu \nu}^{0}+\nabla^{\rho} \nabla_{\mu} h_{\nu \rho}^{0}+\nabla^{\rho} \nabla_{\nu} h_{\mu \rho}^{0}-\nabla_{\mu} \nabla_{\nu} h^{0}\right)$, where $L_{E}$ is the Einstein operator. Therefore, $h_{\mu \nu}^{0}$ is not the correct 4-dimensional graviton field. Neglecting the quadratic terms in equations (2.26)-(2.30), the combination which satisfies the 4dimensional linearized Einstein equation is

$$
\hat{h}_{\mu \nu}^{0} \equiv h_{\mu \nu}^{0}-\frac{1}{4} g_{\mu \nu} \phi^{0}+\frac{1}{24} g_{\mu \nu} \hat{\psi}^{0} .
$$

However, when we take into account the quadratic terms in $(2.26)-(2.30), \hat{h}_{\mu \nu}^{0}$ still does not represent the correct 4-dimensional graviton field. In order to obtain the 4-dimensional graviton field, we need non-trivial field redefinitions to absorb the quadratic terms in the above equations of motion. Such field redefinition will be presented in the next subsection. Now we combine equations (2.26)-(2.30) to obtain the following quadratic equation for $\hat{h}_{\mu \nu}^{0}$ :

$$
\begin{aligned}
\square \hat{h}_{\mu \nu}^{0}= & \nabla^{\rho} \nabla_{\mu} h_{\rho \nu}^{0}+\nabla^{\rho} \nabla_{\nu} h_{\rho \mu}^{0}-\nabla_{\mu} \nabla_{\nu} h^{0}+\frac{1}{2} \nabla_{\mu} \nabla_{\nu} \phi^{0}-\frac{1}{12} \nabla_{\mu} \nabla_{\nu} \hat{\psi}^{0}+\frac{24}{L^{2}} \hat{h}_{\mu \nu}^{0} \\
& +Q_{\mu \nu}^{0}-\frac{L}{12} \tilde{P}_{\mu \nu}^{0}-\frac{1}{2} g_{\mu \nu}\left(Q^{0}-\frac{L}{12} \tilde{P}^{0}\right) .
\end{aligned}
$$

Using the Einstein operator, we write (2.33) as

$$
\left(L_{E}+\frac{12}{L^{2}}\right) \hat{h}_{\mu \nu}^{0}+\frac{1}{2} Q_{\mu \nu}^{0}-\frac{L}{24} \tilde{P}_{\mu \nu}^{0}-\frac{1}{4} g_{\mu \nu}\left(Q^{0}-\frac{L}{12} \tilde{P}^{0}\right)=0
$$

The explicit forms of the quadratic terms $\left(Q_{\mu \nu}^{0}, \tilde{P}_{\mu \nu}^{0}\right.$ etc. $)$ are too long to display here, however, we would like to note that they all contain the terms which are quadratic in the fields $h_{\mu \nu}^{I_{1}}, h^{I_{1}}, \phi^{I_{1}}, u^{I_{1}}, s^{I_{1}}, t_{\mu}^{I_{1}}, t^{I_{35}}$ and their derivatives. In [26, 27] we have obtained the asymptotic expansion of the values of these fields in the LLM solutions. We have shown that, except for the modes with $I_{1}=2$ and $I_{35}=1$, the asymptotic expansions of the other modes, including the zero modes, are nonlinear in the expansion parameter $\mu_{0}$. In particular, the leading terms of all the zero modes that appear in the above equations are quadratic in $\mu_{0}$. Therefore, in order to solve the equations of motion of those zero modes at $\mu_{0}^{2}$ order, the quadratic terms in the above equations are built only by the modes with $I_{1}=2$ and $I_{35}=1$. Having said that, we can simplify (2.34) by using the linearized equations of motion for the $I_{1}=2$ and $I_{35}=1$ modes, which can be read from the list of equations in the previous subsection.

$$
\begin{array}{rlrl}
\square h_{\mu \nu}^{2} & =\frac{40}{L^{2}} h_{\mu \nu}^{2}+\nabla^{\rho} \nabla_{\mu} h_{\rho \nu}^{2}+\nabla^{\rho} \nabla_{\nu} h_{\rho \mu}^{2}-\nabla_{\mu} \nabla_{\nu} h^{2}-\nabla_{\mu} \nabla_{\nu} \phi^{2}-\frac{4}{3 L^{2}} g_{\mu \nu} \hat{\psi}^{2}, \\
\square \phi^{2} & =\frac{100}{L^{2}} h^{2}+\frac{108}{7 L^{2}} \phi^{2}+\frac{1728}{7 L^{4}} s^{2}-\frac{14}{3 L^{2}} u^{2}, & \square \hat{\psi}^{2}=\frac{100}{L^{2}} \hat{\psi}^{2}+\frac{1944}{7 L^{2}} \phi^{2}+\frac{31104}{7 L^{4}} s^{2}, \\
\square s^{2} & =-\frac{80}{7 L^{2}} s^{2}-\frac{5}{7} \phi^{2}-h^{2}, & t_{\mu}^{2}=\frac{54}{7 L} \nabla_{\mu} s^{2}+\frac{6 L}{7} \nabla_{\mu} \phi^{2}-L \nabla^{\nu} h_{\mu \nu}^{2}+L \nabla_{\mu} h^{2}, \\
\square t_{+}^{1} & =-\frac{8}{L^{2}} t_{+}^{1} . & &
\end{array}
$$


Here we picked only $t_{+}^{1}$ from the $t_{ \pm}^{I_{35}=1}$ pair because the leading term in the asymptotic expansion of $t_{-}^{1}$ is cubic in $\mu_{0}$. Then we obtain

$$
\begin{aligned}
& \left(L_{E}+\frac{12}{L^{2}}\right) \hat{h}_{\mu \nu}^{0}+\frac{1}{40}\left\{\frac{3}{8}\left(\nabla_{\mu} \nabla_{\nu} h^{2} h^{2}-\nabla_{\mu} \nabla_{\nu} h^{2} \phi^{2}-h^{2} \nabla_{\mu} \nabla_{\nu} \phi^{2}+\frac{19}{21} \nabla_{\mu} \nabla_{\nu} \phi^{2} \phi^{2}\right)\right. \\
& +\frac{1}{48}\left(-\nabla_{\mu} \nabla_{\nu} h^{2} u^{2}-h^{2} \nabla_{\mu} \nabla_{\nu} u^{2}-\nabla_{\mu} h^{2} \nabla_{\nu} u^{2}-\nabla_{\nu} h^{2} \nabla_{\mu} u^{2}+\nabla_{\mu} \nabla_{\nu} \phi^{2} u^{2}\right. \\
& \left.+\phi^{2} \nabla_{\mu} \nabla_{\nu} u^{2}+\nabla_{\mu} \phi^{2} \nabla_{\nu} u^{2}+\nabla_{\nu} \phi^{2} \nabla_{\mu} u^{2}\right)+\frac{11}{72} \nabla_{\mu} h^{2} \nabla_{\nu} h^{2}+\frac{55}{392} \nabla_{\mu} \phi^{2} \nabla_{\nu} \phi^{2} \\
& -\frac{3672}{49 L^{4}} \nabla_{\mu} s^{2} \nabla_{\nu} s^{2}-\frac{95}{168}\left(\nabla_{\mu} h^{2} \nabla_{\nu} \phi^{2}+\nabla_{\nu} h^{2} \nabla_{\mu} \phi^{2}\right)-\frac{12}{7 L^{2}}\left(\nabla_{\mu} h^{2} \nabla_{\nu} s^{2}+\nabla_{\nu} h^{2} \nabla_{\mu} s^{2}\right) \\
& -\frac{72}{49 L^{2}}\left(\nabla_{\mu} \phi^{2} \nabla_{\nu} s^{2}+\nabla_{\nu} \phi^{2} \nabla_{\mu} s^{2}\right)-\frac{2}{9} \nabla^{\rho} h_{\mu \rho}^{2} \nabla^{\sigma} h_{\sigma \nu}^{2}+\frac{2}{9}\left(\nabla_{\mu} h^{2} \nabla^{\rho} h_{\rho \nu}^{2}+\nabla_{\nu} h^{2} \nabla^{\rho} h_{\mu \rho}^{2}\right) \\
& +\frac{4}{21}\left(\nabla_{\mu} \phi^{2} \nabla^{\rho} h_{\rho \nu}^{2}+\nabla_{\nu} \phi^{2} \nabla^{\rho} h_{\mu \rho}^{2}\right)+\frac{12}{7 L^{2}}\left(\nabla_{\mu} s^{2} \nabla^{\rho} h_{\rho \nu}^{2}+\nabla_{\nu} s^{2} \nabla^{\rho} h_{\mu \rho}^{2}\right)+\nabla_{\mu} h^{2 \rho \sigma} \nabla_{\nu} h_{\rho \sigma}^{2} \\
& -\frac{1}{2} \nabla_{\rho}\left(h^{2 \rho \sigma} \nabla_{\mu} h_{\sigma \nu}^{2}+h^{2 \rho \sigma} \nabla_{\nu} h_{\sigma \mu}^{2}\right)+\frac{1}{2} \nabla_{\rho}\left(h^{2 \rho \sigma} \nabla_{\sigma} h_{\mu \nu}^{2}\right)-\frac{1}{2} \nabla^{\rho} \nabla^{\sigma} h_{\rho \sigma}^{2} h_{\mu \nu}^{2}-\frac{216}{7 L^{4}} s^{2} \nabla_{\mu} \nabla_{\nu} s^{2} \\
& +\frac{5}{4} h^{2 \rho \sigma} \nabla_{\nu} \nabla_{\mu} h_{\rho \sigma}^{2}+\frac{1}{4} \nabla^{\rho}\left(h^{2}+\phi^{2}\right)\left(\nabla_{\mu} h_{\rho \nu}^{2}+\nabla_{\nu} h_{\rho \mu}^{2}\right)-\frac{1}{4} \nabla^{\rho}\left(h^{2}+\phi^{2}\right) \nabla_{\rho} h_{\mu \nu}^{2}+\frac{23}{L^{2}} h^{2} h_{\mu \nu}^{2} \\
& -\frac{1}{2} \nabla^{\sigma} h_{\rho \nu}^{2} \nabla^{\rho} h_{\sigma \mu}^{2}+\frac{1}{2} \nabla^{\sigma} h_{\nu}^{2 \rho} \nabla_{\sigma} h_{\mu \rho}^{2}+\frac{8}{L^{2}} h_{\nu}^{2 \rho} h_{\mu \rho}^{2}+\frac{17}{2 L^{2}} g_{\mu \nu} h^{2 \rho \sigma} h_{\rho \sigma}^{2}+\frac{1}{2} g_{\mu \nu} \nabla_{\rho}\left(h^{2 \rho \sigma} \nabla_{\sigma} \phi^{2}\right) \\
& +\frac{3}{16} g_{\mu \nu} \nabla^{\rho} h^{2} \nabla_{\rho} h^{2}-\frac{1}{2} g_{\mu \nu} \nabla^{\rho} h^{2} \nabla_{\rho} \phi^{2}+\frac{5}{112} g_{\mu \nu} \nabla^{\rho} \phi^{2} \nabla_{\rho} \phi^{2}-\frac{201}{98 L^{2}} g_{\mu \nu} \phi^{2} \phi^{2}-\frac{40}{7 L^{2}} g_{\mu \nu} \phi^{2} h^{2} \\
& -\frac{33}{L^{2}} g_{\mu \nu} h^{2} h^{2}-\frac{1}{7 L^{2}} h_{\mu \nu}^{2} \phi^{2}+\frac{432}{7 L^{4}} h_{\mu \nu}^{2} s^{2}+\frac{1}{2} h_{\mu \nu}^{2} \square h^{2}+\frac{3}{4} g_{\mu \nu} h^{2 \rho \sigma} \nabla^{\tau} \nabla_{\rho} h_{\tau \sigma}^{2}+\frac{26352}{49 L^{6}} g_{\mu \nu} s^{2} s^{2} \\
& -\frac{108}{49 L^{4}} g_{\mu \nu} \phi^{2} s^{2}-\frac{11}{6 L^{2}} h_{\mu \nu}^{2} u^{2}-\frac{1}{18 L^{2}} g_{\mu \nu} u^{2} u^{2}+\frac{25}{8 L^{2}} g_{\mu \nu} h^{2} u^{2}-\frac{1}{2} g_{\mu \nu}\left(\frac{3}{4} h^{2 \rho \sigma} \nabla_{\rho} \nabla_{\sigma} h^{2}\right. \\
& -\frac{1}{2} \nabla^{\rho} \nabla^{\sigma} h_{\rho \sigma}^{2} h^{2}+\frac{5}{4} h^{2 \rho \sigma} \nabla_{\rho} \nabla_{\sigma} \phi^{2}-\frac{3}{4} \nabla^{\lambda} h^{2 \rho \sigma} \nabla_{\lambda} h_{\rho \sigma}^{2}+\frac{216}{7 L^{4}} \nabla^{\rho} s^{2} \nabla_{\rho} s^{2}+\frac{1}{2} h^{2} \square h^{2} \\
& \left.+\frac{1}{2} \nabla^{\rho} \phi^{2} \nabla^{\sigma} h_{\rho \sigma}^{2}-\frac{46}{56 L^{2}} \phi^{2} u^{2}+\frac{216}{7 L^{4}} h^{2} s^{2}-\frac{36}{7 L^{4}} u^{2} s^{2}\right)-\frac{1}{48} g_{\mu \nu}\left(\frac{1}{2} \square h^{2} u^{2}+\nabla^{\lambda} h^{2} \nabla_{\lambda} u^{2}\right. \\
& \left.\left.-\nabla^{\lambda} \phi^{2} \nabla_{\lambda} u^{2}\right)\right\}+\frac{1}{48}\left(\nabla_{\mu} \nabla_{\nu} t_{+}^{1} t_{+}^{1}+\frac{1}{2} \nabla_{\mu} t_{+}^{1} \nabla_{\nu} t_{+}^{1}\right)+\frac{1}{96} g_{\mu \nu}\left(\nabla_{\rho} t_{+}^{1} \nabla^{\rho} t_{+}^{1}-\frac{16}{L^{2}} t_{+}^{1} t_{+}^{1}\right)=0 .
\end{aligned}
$$

In our case, the fluctuation modes $\left(h_{\mu \nu}^{I_{1}}, \phi^{I_{1}}\right.$, etc.) represent the deviations of the LLM geometry from the $\mathrm{AdS}_{4} \times S^{7}$ space. In that case, we have shown that the symmetrizedtraceless transverse KK graviton mode $\check{h}_{(\mu \nu)}^{2}$, which is given by [26, 27]

$$
\check{h}_{(\mu \nu)}^{2}=\hat{\phi}_{(\mu \nu)}^{2}+\frac{7}{30} \hat{\psi}_{(\mu \nu)}^{2}+\frac{L^{2}}{8} \nabla_{(\mu} \nabla_{\nu)} \hat{\phi}^{2}-\frac{7 L^{2}}{720} \nabla_{(\mu} \nabla_{\nu)} \hat{\psi}^{2}
$$

with $\hat{\psi}_{\mu \nu}^{2} \equiv 18 h_{\mu \nu}^{2}-\frac{L}{2}\left(\nabla_{\mu} t_{\nu}^{2}+\nabla_{\nu} t_{\mu}^{2}\right), \hat{\phi}_{\mu \nu}^{2} \equiv-\frac{7}{5}\left(h_{\mu \nu}^{2}+\nabla_{\mu} \nabla_{\nu} s^{2}\right), \hat{\phi}^{2} \equiv \phi^{2}+\frac{16}{L^{2}} s^{2}$, is vanishing at linear order in $\mu_{0}$. This implies, at linear order in $\mu_{0}, h_{\mu \nu}^{2}$ is not an independent tensor mode and can be expressed in terms of the scalar modes as

$$
h_{\mu \nu}^{2}=-\frac{L^{2}}{576} \nabla_{\mu} \nabla_{\nu} \hat{\psi}^{2}+\frac{11 L^{2}}{224} \nabla_{\mu} \nabla_{\nu} \phi^{2}-\frac{3}{14} \nabla_{\mu} \nabla_{\nu} s^{2}-g_{\mu \nu}\left(\frac{1}{72} \hat{\psi}^{2}+\frac{3}{28} \hat{\phi}^{2}\right) .
$$


We have also shown that, only two gauge invariant combinations of the four scalar fields $h^{2}, \phi^{2}, u^{2}, s^{2}$ are physical modes. The two gauge invariant physical scalar modes are $\hat{\psi}^{2}$ and $\hat{\phi}^{2}$ so that we express the four scalars as

$$
h^{2}=a_{1} \hat{\psi}^{2}+b_{1} \hat{\phi}^{2}, \quad u^{2}=a_{2} \hat{\psi}^{2}+b_{2} \hat{\phi}^{2}, \quad \phi^{2}=a_{3} \hat{\psi}^{2}+b_{3} \hat{\phi}^{2}, \quad s^{2}=a_{4} \hat{\psi}^{2}+b_{4} \hat{\phi}^{2} .
$$

In particular, these relations should be valid when we substitute the values of those scalar fields from the asymptotic expansion of the LLM solutions. In that case we find the following relations among the constants $a_{i}$ and $b_{i}$,

$$
b_{1}=-\frac{3}{7}\left(1-6 a_{1}\right), \quad b_{2}=-\frac{18}{7}\left(2-a_{2}\right), \quad b_{3}=\frac{3}{7}\left(1+6 a_{3}\right), \quad b_{4}=\frac{L^{2}}{28}\left(1+\frac{72 a_{4}}{L^{2}}\right) .
$$

It is more convenient to use the diagonal modes $\check{\phi}^{2}=\frac{9}{70}\left(7 \hat{\psi}^{2}+18 \hat{\phi}^{2}\right), \check{\psi}^{2}=\frac{1}{70}\left(7 \hat{\psi}^{2}-162 \hat{\phi}^{2}\right)$. See $[26,27]$ for the derivation of these diagonal modes. Then we can write

$$
\begin{aligned}
h^{2}=\frac{1}{6} \check{\psi}^{2}+\frac{1}{9}\left(10 a_{1}-\frac{1}{6}\right) \check{\phi}^{2}, & u^{2}=2 \check{\psi}^{2}+\frac{2}{9}\left(5 a_{2}-1\right) \check{\phi}^{2}, \\
\phi^{2}=-\frac{1}{6} \check{\psi}^{2}+\frac{1}{9}\left(10 a_{3}+\frac{1}{6}\right) \check{\phi}^{2}, & s^{2}=-\frac{L^{2}}{72} \check{\psi}^{2}+\frac{1}{9}\left(10 a_{4}+\frac{L^{2}}{72}\right) \check{\phi}^{2} .
\end{aligned}
$$

In the LLM solution, the asymptotic expansion of $\check{\phi}^{2}$ is of order $\mu_{0}^{3}$ and it does not contribute to the equations of motion at $\mu_{0}^{2}$ order. Therefore, we can set $\check{\phi}^{2}$ to zero and use (2.38) and (2.41) in (2.36) to write the quadratic part of the equation of motion only in terms of $\check{\psi}^{2}$ as follows

$$
\begin{aligned}
& \left(L_{E}+\frac{12}{L^{2}}\right) \hat{h}_{\mu \nu}^{0}+\frac{1}{34560}\left\{-\frac{26}{3} \nabla_{\mu} \check{\psi}^{2} \nabla_{\nu} \check{\psi}^{2}+\frac{28}{3} \check{\psi}^{2} \nabla_{\mu} \nabla_{\nu} \check{\psi}^{2}+\frac{L^{2}}{3} \nabla_{\mu} \nabla^{\rho} \check{\psi}^{2} \nabla_{\nu} \nabla_{\rho} \check{\psi}^{2}\right. \\
& +\frac{L^{2}}{2} \nabla^{\rho} \check{\psi}^{2} \nabla_{\mu} \nabla_{\nu} \nabla_{\rho} \check{\psi}^{2}+\frac{L^{4}}{24} \nabla_{\mu} \nabla^{\rho} \nabla^{\sigma} \check{\psi}^{2} \nabla_{\nu} \nabla_{\rho} \nabla_{\sigma} \check{\psi}^{2}+\frac{L^{4}}{32} \nabla^{\rho} \nabla^{\sigma} \check{\psi}^{2} \nabla_{\mu} \nabla_{\nu} \nabla_{\rho} \nabla_{\sigma} \check{\psi}^{2} \\
& \left.-g_{\mu \nu}\left(\frac{12}{L^{2}} \check{\psi}^{2} \check{\psi}^{2}+\nabla^{\rho} \check{\psi}^{2} \nabla_{\rho} \check{\psi}^{2}+\frac{35 L^{2}}{48} \nabla^{\rho} \nabla^{\sigma} \check{\psi}^{2} \nabla_{\rho} \nabla_{\sigma} \check{\psi}^{2}-\frac{L^{4}}{64} \nabla^{\tau} \nabla^{\rho} \nabla^{\sigma} \breve{\psi}^{2} \nabla_{\tau} \nabla_{\rho} \nabla_{\sigma} \check{\psi}^{2}\right)\right\} \\
& +\frac{1}{48}\left(\nabla_{\mu} \nabla_{\nu} t_{+}^{1} t_{+}^{1}+\frac{1}{2} \nabla_{\mu} t_{+}^{1} \nabla_{\nu} t_{+}^{1}\right)+\frac{1}{96} g_{\mu \nu}\left(\nabla_{\rho} t_{+}^{1} \nabla^{\rho} t_{+}^{1}-\frac{16}{L^{2}} t_{+}^{1} t_{+}^{1}\right)=0 .
\end{aligned}
$$

In the next subsection we introduce some non-trivial field redefinitions in order to eliminate the higher derivative terms in the above equation and obtain a linearized equation for the 4-dimensional fields.

\subsection{The KK mapping at quadratic order}

Our goal in this section is to apply the KK reduction procedure to the 11-dimensional supergravity on LLM geometry and construct 4-dimensional gravity theory whose solution encodes the information about the asymptotic limit of the LLM geometry. In the previous subsection, using the graviton mode example in (2.42), we have shown that the compactification of 11-dimensional supergravity on $S^{7}$ results in the field equations which contain higher derivative terms. In general, the same is true for all the modes in the KK towers. 
To absorb the higher derivative terms we need to introduce some field redefinitions. For instance, for some scalar KK mode whose equation of motion contains up to four derivatives, the field redefinition is of the form

$$
S^{I}=s^{I}+K_{I J_{1} J_{2}} t^{J_{1}} t^{J_{2}}+L_{I J_{1} J_{2}} \nabla_{\mu} t^{J_{1}} \nabla^{\mu} t^{J_{2}},
$$

where $K_{I J_{1} J_{2}}, L_{I J_{1} J_{2}}$ are some numerical coefficients, $s^{I}$ represents a gauge invariant 11dimensional field and $S^{I}$ is the corresponding 4-dimensional field. See [28] for a systematic procedure of the KK reduction. The $t^{J_{i}}$ 's represent all the fields that appear in higher derivative part of the equations of motion of $s^{I}$. If the field equation contains more than four derivatives, then the field redefinition will contain more than two derivatives. For the important scalar modes $\check{\psi}^{2}$ and $t_{+}^{1}$ discussed in the previous subsection, the equations of motion involve higher derivative terms only if we want to solve them at cubic or higher order in $\mu_{0}$. Up to quadratic order, their equations of motion are linear and are given by

$$
\left(\square-M_{t}^{2}\right) t_{+}^{1}=0, \quad\left(\square-M_{\psi}^{2}\right) \check{\psi}^{2}=0,
$$

where $M_{t}^{2}=M_{\psi}^{2}=-\frac{8}{L^{2}}$. In this case, the field redefinitions are trivial and we can write the corresponding 4-dimensional fields as,

$$
\Psi=\check{\psi}^{2}, \quad T=t_{+}^{1} .
$$

The 4-dimensional gravity action with matter, which yields the equations of motion in (2.44) for $\Psi$ and $T$ is given by

$$
S=\frac{1}{16 \pi G_{N}^{(4)}} \int d^{4} x \sqrt{-g}(\hat{R}-2 \Lambda)+S_{m},
$$

where $\Lambda=-\frac{(d-1)(d-2)}{2 L_{\mathrm{AdS}_{4}}^{2}}=-\frac{12}{L^{2}}$ and

$$
S_{m}=-\frac{A_{t}}{2} \int d^{4} x \sqrt{-g}\left(\nabla_{\mu} T \nabla^{\mu} T+M_{t}^{2} T^{2}\right)-\frac{A_{\psi}}{2} \int d^{4} x \sqrt{-g}\left(\nabla_{\mu} \Psi \nabla^{\mu} \Psi+M_{\psi}^{2} \Psi^{2}\right),
$$

for some over all normalizations $A_{t}$ and $A_{\psi}$ which will be fixed later. The corresponding energy-momentum tensor is

$$
\begin{aligned}
\tilde{T}_{\mu \nu}=-\frac{2}{\sqrt{-g}} \frac{\delta S_{m}}{\delta g^{\mu \nu}}= & A_{t}\left[\nabla_{\mu} T \nabla_{\nu} T-\frac{1}{2} g_{\mu \nu}\left(\nabla_{\rho} T \nabla^{\rho} T+M_{t}^{2} T^{2}\right)\right] \\
& +A_{\psi}\left[\nabla_{\mu} \Psi \nabla_{\nu} \Psi-\frac{1}{2} g_{\mu \nu}\left(\nabla_{\rho} \Psi \nabla^{\rho} \Psi+M_{\psi}^{2} \Psi^{2}\right)\right] .
\end{aligned}
$$

The next step is to obtain the equation of motion for the 4-dimensional graviton by using the result in (2.42) and the above energy-momentum tensor. Since the background is $\mathrm{AdS}_{4}$, we consider the 4-dimensional Einstein equation with negative cosmological constant

$$
\hat{R}_{\mu \nu}-\frac{1}{2} \hat{g}_{\mu \nu} \hat{R}+\Lambda \hat{g}_{\mu \nu}=8 \pi G_{N} \tilde{T}_{\mu \nu}
$$


where $\hat{g}_{\mu \nu}=g_{\mu \nu}+\delta g_{\mu \nu}$ represents the metric which is deviated from the $\mathrm{AdS}_{4}$ due to the presence of the energy-momentum tensor of the matter fields $\Psi$ and $T$. In order to obtain the equations for the graviton field, we insert the perturbed metric into (2.49) and keep only up to the terms that are linear in the fluctuations

$$
\delta R_{\mu \nu}-\frac{1}{2} \delta g_{\mu \nu} R-\frac{1}{2} g_{\mu \nu} \delta g^{\rho \sigma} R_{\rho \sigma}-\frac{1}{2} g_{\mu \nu} g^{\rho \sigma} \delta R_{\rho \sigma}-\frac{12}{L^{2}} \delta g_{\mu \nu}=8 \pi G_{N} \tilde{T}_{\mu \nu} .
$$

Setting $\delta g_{\mu \nu}=H_{\mu \nu}, \delta g^{\mu \nu}=-H^{\mu \nu}$, we can write

$$
\begin{aligned}
& \frac{1}{2}\left(-\square H_{\mu \nu}+\nabla^{\rho} \nabla_{\mu} H_{\rho \nu}+\nabla^{\rho} \nabla_{\nu} H_{\rho \mu}-\nabla_{\mu} \nabla_{\nu} H\right)+\frac{12}{L^{2}} H_{\mu \nu}-\frac{6}{L^{2}} g_{\mu \nu} H \\
& \quad-\frac{1}{2} g_{\mu \nu}\left(\nabla^{\rho} \nabla^{\sigma} H_{\rho \sigma}-\square H\right)=8 \pi G_{N} \tilde{T}_{\mu \nu} .
\end{aligned}
$$

Taking the trace, we obtain

$$
-\frac{12}{L^{2}} H-\nabla^{\rho} \nabla^{\sigma} H_{\rho \sigma}+\square H=8 \pi G_{N} g^{\rho \sigma} \tilde{T}_{\rho \sigma} .
$$

Plugging this into (2.51) and using the energy-momentum tensor in (2.48), we obtain

$$
\begin{aligned}
\left(L_{E}+\frac{12}{L^{2}}\right) H_{\mu \nu} & -8 \pi G_{N} A_{t}\left(\nabla_{\mu} T \nabla_{\nu} T+\frac{M_{t}^{2}}{2} g_{\mu \nu} T^{2}\right) \\
& -8 \pi G_{N} A_{\psi}\left(\nabla_{\mu} \Psi \nabla_{\nu} \Psi+\frac{M_{\psi}^{2}}{2} g_{\mu \nu} \Psi^{2}\right)=0 .
\end{aligned}
$$

The final step is to introduce a field redefinition which eliminates the higher derivative terms in (2.42) and reduces it to (2.53). Since the equation of motion in (2.42) contains up to six derivative terms in $\check{\psi}^{2}$ and no higher derivative term in $t_{+}^{1}$, the field redefinition should contain up to four derivative terms in $\check{\psi}^{2}$ and no derivative in $t_{+}^{1}$. The required field redefinition is given by

$$
\begin{aligned}
H_{\mu \nu}= & \hat{h}_{\mu \nu}^{0}+g_{\mu \nu}\left(\tilde{C}_{1} \check{\psi}^{2} \check{\psi}^{2}+\tilde{C}_{2} \nabla^{\rho} \check{\psi}^{2} \nabla_{\rho} \check{\psi}^{2}\right)+\tilde{C}_{3} \nabla_{\mu} \check{\psi}^{2} \nabla_{\nu} \check{\psi}^{2} \\
& +g_{\mu \nu} \tilde{C}_{4} \nabla^{\rho} \nabla^{\sigma} \check{\psi}^{2} \nabla_{\rho} \nabla_{\sigma} \check{\psi}^{2}+\tilde{C}_{5} \nabla_{\mu} \nabla^{\rho} \check{\psi}^{2} \nabla_{\nu} \nabla_{\rho} \check{\psi}^{2}+g_{\mu \nu} \tilde{C}_{t} t_{+}^{1} t_{+}^{1}
\end{aligned}
$$

Inserting (2.54) into (2.53) and comparing the result with (2.42), we determine the unknown coefficients listed below,

$$
\begin{array}{llrl}
\tilde{C}_{1}=-\frac{1}{2^{6} 3^{3} 5}, & \tilde{C}_{2}=-\frac{L^{2}}{2^{11} 3^{3} 5}, & \tilde{C}_{3}=-\frac{7 L^{2}}{2^{11} 3^{4} 5}, & \tilde{C}_{4}=-\frac{L^{4}}{2^{14} 3^{3} 5}, \\
\tilde{C}_{5}=-\frac{L^{4}}{2^{13} 3^{4} 5}, & \tilde{C}_{t}=-\frac{1}{2^{5} 3}, & 8 \pi G_{N} A_{t}=\frac{1}{2^{5} 3}, & 8 \pi G_{N} A_{\psi}=\frac{1}{2^{8} 3^{2}} .
\end{array}
$$

In this paper we focused on obtaining the field redefinition for the graviton mode. A similar procedure determines the field redefinition for higher KK tensor, vector, and scalar modes in 4-dimension. In $[28,29]$ a similar result was obtained for 5-dimensional KK towers, which are obtained from the dimensional reduction of the 10-dimensional type-IIB supergravity on background which is asymptotically $\mathrm{AdS}_{5} \times S^{5}$. The resulting field redefinition was dubbed the KK map between the 10-dimensional and 5-dimensional fields. In our case, the KK map for graviton field is as in (2.54). 


\section{HEE from vev and source}

When the conformal symmetry is broken due to a relevant deformation of a CFT, the CPOs develop non-vanishing one-point functions. In that case, one can infer that the deviation of the EE from it's value in the CFT is related to the non-vanishing one-point function. From the perspective of the dual gravity, the non-conformal field theory corresponds to a gravity theory on an asymptotically AdS geometry. According to the RT conjecture, the deviation of the geometry from the AdS space induces a variation in HEE. In other words, non-vanishing one-point function of CPO of gauge theory induces the variation of $\mathrm{EE}$ and induces change of geometry in dual gravity. The dynamics of the geometry is governed by the linearized Einstein equations with matter fields interactions on the AdS background. Understanding the field theory counterpart of these governing equations is intriguing. Research in this direction is progressing. In this paper, we move further this progress by studying the HEE in mABJM theory.

In $[26,27]$, we presented the calculation of one-point function of the CPO with conformal dimension one as an evidence supporting the duality between the mABJM theory and 11-dimensional supergravity theory on the LLM geometries. We obtained the onepoint function, $\left\langle\mathcal{O}^{(1)}\right\rangle$ in the large $N$ limit, using the KK holography method from the 11-dimensional LLM geometry and showed that there is an exact agreement with the results obtained by using the supersymmetric vacua of mABJM theory. We conducted this test for all supersymmetric vacua, which are infinite in number. In order to strengthen the confirmation of the duality of the two theories, those results will be extended to the case of conformal dimension two CPO [31]. In this paper, we exploit this exact correspondence to study the HEE based on the RT conjecture. To that end, we start by reading the asymptotically AdS solutions of the 4-dimensional gravity theory, which are constructed in the previous section, from the 11-dimensional LLM solutions. Applying the RT conjecture to those 4-dimensional gravity solutions, we calculate the leading order deviation of the HEE $(\delta S)$ from its value in pure $\mathrm{AdS}_{4}$ space. We compare the result with the known result of the HEE in the LLM geometry [46].

\subsection{Vacua of mABJM theory and LLM geometries}

Under the supersymmetry preserving mass deformation of the ABJM theory, the global $\mathrm{SU}(4)$ symmetry of the ABJM theory is broken to $\mathrm{SU}(2) \times \mathrm{SU}(2) \times \mathrm{U}(1)$. To express the vacuum solution which reflects the broken symmetry, we split the scalar fields into $Y^{A}=$ $\left(Z^{a}, W^{\dagger a}\right)$, where $A=1,2,3,4$ and $a=1,2$. One interesting feature of the mABJM theory is that it has discrete Higgs vacua, which are represented as direct sums of GRVV matrices [23]. The vacua are classified by occupation numbers, $N_{n}$ and $N_{n}^{\prime}$ which are respectively denote the numbers of $n \times(n+1)$ GRVV matrices $\mathcal{M}_{n}$ and $(n+1) \times n$ GRVV matrices $\overline{\mathcal{M}}_{n}$, in the direct sums. See [50] for the details. These vacua are supersymmetric if the occupation numbers are in the range, $0 \leq N_{n}, N_{n}^{\prime} \leq k$ [52]. There is a one-to-one map between the discrete Higgs vacua of the mABJM theory and the LLM geometries with $\mathbb{Z}_{k}$ orbifold, which have $\mathrm{SO}(2,1) \times \mathrm{SO}(4) / \mathbb{Z}_{k} \times \mathrm{SO}(4) / \mathbb{Z}_{k}$ isometry. 
The LLM metric and the corresponding 4-form field strength are given by

$$
\begin{aligned}
d s^{2}= & -\mathbf{G}_{t t}\left(-d t^{2}+d w_{1}^{2}+d w_{2}^{2}\right)+\mathbf{G}_{x x}\left(d \tilde{x}^{2}+d \tilde{y}^{2}\right)+\mathbf{G}_{\theta \theta} d s_{S^{3} / \mathbb{Z}_{k}}^{2}+\mathbf{G}_{\tilde{\theta} \tilde{\theta}} d s_{\tilde{S}^{3} / \mathbb{Z}_{k}}^{2}, \\
\mathbf{F}_{4}= & -d\left(e^{2 \Phi} h^{-2} V\right) \wedge d t \wedge d w_{1} \wedge d w_{2}+\mu_{0}^{-1}\left[V d\left(\tilde{y}^{2} e^{2 G}\right)+h^{2} e^{3 G} \star_{2} d\left(\tilde{y}^{2} e^{-2 G}\right)\right] \wedge d \Omega_{3} \\
& +\mu_{0}^{-1}\left[V d\left(\tilde{y}^{2} e^{-2 G}\right)-h^{2} e^{-3 G} \star_{2} d\left(\tilde{y}^{2} e^{2 G}\right)\right] \wedge d \tilde{\Omega}_{3},
\end{aligned}
$$

where $\mu_{0}$ is a mass parameter, $d s_{S^{3} / \mathbb{Z}_{k}}^{2}$ and $d s_{\tilde{S}^{3} / \mathbb{Z}_{k}}^{2}$ are line elements of three-spheres with $\mathbb{Z}_{k}$ orbifold, and $d \Omega_{3}=-(\sin \theta / 8) d \theta \wedge d \phi \wedge d \psi, d \tilde{\Omega}_{3}=-(\sin \tilde{\theta} / 8) d \tilde{\theta} \wedge d \tilde{\phi} \wedge d \tilde{\psi}$ are the volume forms of the two spheres in the Euler coordinate system. The warp factors and the functions defining the 4 -form field strength are given by

$$
\begin{aligned}
& \mathbf{G}_{t t}=-\left(\frac{4 \mu_{0}^{2} \tilde{y} \sqrt{\frac{1}{4}-Z^{2}}}{f^{2}}\right)^{2 / 3}, \quad \mathbf{G}_{x x}=\left(\frac{f \sqrt{\frac{1}{4}-Z^{2}}}{2 \mu_{0} \tilde{y}^{2}}\right)^{2 / 3}, \quad \mathbf{G}_{\theta \theta}=\left(\frac{f \tilde{y} \sqrt{\frac{1}{2}+Z}}{2 \mu_{0}\left(\frac{1}{2}-Z\right)}\right)^{2 / 3}, \\
& \mathbf{G}_{\tilde{\theta} \tilde{\theta}}=\left(\frac{f \tilde{y} \sqrt{\frac{1}{2}-Z}}{2 \mu_{0}\left(\frac{1}{2}+Z\right)}\right)^{2 / 3}, \quad h^{2}=\frac{\sqrt{\frac{1}{4}-Z^{2}}}{\tilde{y}}, \quad e^{2 \Phi}=\frac{4 \tilde{y} \mu_{0}^{2} \sqrt{\frac{1}{4}-Z^{2}}}{f^{2}}, \quad e^{2 G}=\frac{\frac{1}{2}+Z}{\frac{1}{2}-Z}
\end{aligned}
$$

with $f(\tilde{x}, \tilde{y})=\sqrt{1-4 Z^{2}-4 \tilde{y}^{2} V^{2}}$.

Here we notice that the LLM geometry is completely determined by two functions,

$$
Z(\tilde{x}, \tilde{y})=\sum_{i=1}^{2 N_{B}+1} \frac{(-1)^{i+1}\left(\tilde{x}-\tilde{x}_{i}\right)}{2 \sqrt{\left(\tilde{x}-\tilde{x}_{i}\right)^{2}+\tilde{y}^{2}}}, \quad V(\tilde{x}, \tilde{y})=\sum_{i=1}^{2 N_{B}+1} \frac{(-1)^{i+1}}{2 \sqrt{\left(\tilde{x}-\tilde{x}_{i}\right)^{2}+\tilde{y}^{2}}},
$$

where $\tilde{x}_{i}$ 's are numerical parameters. The function $Z(\tilde{x}, \tilde{y})$ at $\tilde{y}=0$ has a value $\frac{1}{2}$ for $\tilde{x}_{2 i-1}<\tilde{x}<\tilde{x}_{2 i}$ and it is $-\frac{1}{2}$ for $\tilde{x}_{2 i}<\tilde{x}<\tilde{x}_{2 i+1}$. The geometries are classified by those values of $Z(\tilde{x}, 0)$. That is, the LLM geometries are represented as an infinite strip in the $\tilde{x}$-direction with $Z(\tilde{x}, 0)=-\frac{1}{2}$ denoted by black strip and $Z(\tilde{x}, 0)=\frac{1}{2}$ denoted by white strip. This representation of the LLM geometry is called the droplet picture. So the $\tilde{x}_{i}$ 's denote the positions of the boundaries between the black and the white strips and $N_{B}$ is the number of finite-sized black/white strips in the droplet representation. Due to flux quantization condition of the 4 -form field strength, the difference between consecutive $\tilde{x}_{i}$ 's is quantized as [50]

$$
\tilde{x}_{i+1}-\tilde{x}_{i}=2 \pi l_{\mathrm{P}}^{3} \mu_{0} \mathbb{Z},
$$

where $l_{\mathrm{P}}$ is the Planck length. Therefore, all LLM geometries are completely determined by these quantized locus $\tilde{x}_{i}$ 's.

In order to consider the gauge/gravity duality near the UV fixed point of the field theory, we need to expand the dual geometry in the asymptotic region. For the asymptotic expansion of the general LLM geometries, it is convenient to introduce new parameters [46],

$$
C_{p}=\sum_{i=1}^{\infty}(-1)^{i+1}\left(\frac{\tilde{x}_{i}}{2 \pi l_{\mathrm{P}}^{3} \mu_{0} \sqrt{A}}\right)^{p}
$$


where $A$ is defined as ${ }^{5}$

$$
A=k N-\frac{1}{2} \sum_{n=0}^{\infty}\left[l_{n}\left(k-l_{n}\right)+l_{n}^{\prime}\left(k-l_{n}^{\prime}\right)\right] .
$$

Here we introduce a new set of parameters, $\left\{l_{n}, l_{n}^{\prime}\right\}$, which are called discrete torsions and used to classify the LLM geometries in the droplet picture. See [50] for the details. The one-to-one correspondence between the vacua of the mABJM theory and the LLM geometries identifies $\left\{N_{n}, N_{n}^{\prime}\right\}$ with $\left\{l_{n}, l_{n}^{\prime}\right\}$.

\subsection{KK reduction of the LLM geometries}

In the Fefferman-Graham coordinate system, the LLM metric is given by (see [26, 27] for details)

$$
\begin{aligned}
d s^{2}= & \frac{L^{2}}{4 z^{2}}\left[d z^{2}+\frac{4 z^{2}}{L^{2}}\left[1+\tilde{g}_{1}(z, \tau)\right]\left(-d t^{2}+d w_{1}^{2}+d w_{2}^{2}\right)\right] \\
& +\left[1+\tilde{g}_{2}(z, \tau)\right] d \tau^{2}+\left[1+\tilde{g}_{3}(z, \tau)\right] d s_{S^{3}}^{2}+\left[1+\tilde{g}_{4}(z, \tau)\right] d s_{\tilde{S}^{3}}^{2},
\end{aligned}
$$

where the $\tilde{g}_{i}(z, \tau)$ represents the deviation of the LLM metric from the $\mathrm{AdS}_{4} \times S^{7}$ background. Similarly, the 4 -form field strength can be split into the background and the rest. In the asymptotic region, these deviations become small fluctuations, and the LLM solution can be written as in (2.3), where the values of the small fluctuations $h_{p q}$ and $f_{p q r s}$ are read from the asymptotic expansion of $\tilde{g}_{i}(z, \tau)$, and the similar terms in the 4 -form field strength. Then, these small fluctuations can be expanded in terms of the spherical harmonics on $S^{7}$, in order to obtain the values of the KK modes $\left(h_{\mu \nu}^{I_{1}}, \phi^{I_{1}}\right.$, etc.) of the previous section. In $[26,27]$, we have listed the full result for all the KK modes up to $\mu_{0}^{2}$ order. Here, we are interested only in the graviton mode, which corresponds to a combination of the KK zero modes. We also need $\left(I_{1}=2, I_{35}=1\right)$ modes, which appear in the quadratic part of the graviton equation of motion. Therefore, we copy the following results from $[26,27]$

$$
\begin{array}{rlrl}
h_{i j}^{0} & =\left[\frac{L^{2} \mu_{0}^{2}}{720}\left(360+7 \beta_{3}^{2}\right)+\mathcal{O}\left(\mu_{0}^{4}\right)\right] \eta_{i j}, & h_{z z}^{0}=0, \\
h^{0}=\frac{\left(\mu_{0} z\right)^{2}}{60}\left(360+7 \beta_{3}^{2}\right)+\mathcal{O}\left(\mu_{0}^{4}\right), & \phi^{0}=\frac{\left(\mu_{0} z\right)^{2}}{15}\left(-80+\beta_{3}^{2}\right)+\mathcal{O}\left(\mu_{0}^{4}\right), \\
\hat{\psi}^{0} & =\frac{6\left(\mu_{0} z\right)^{2}}{5}\left(80+\beta_{3}^{2}\right)+\mathcal{O}\left(\mu_{0}^{4}\right), & \check{\psi}^{2}=-24 \beta_{3} \mu_{0} z+\mathcal{O}\left(\mu_{0}^{3}\right), \\
t_{+}^{1}=16 \sqrt{3} \mu_{0} z+\mathcal{O}\left(\mu_{0}^{3}\right), & &
\end{array}
$$

where $\eta_{i j}=\operatorname{diag}(-1,1,1)$ and

$$
\beta_{3}=2 C_{1}^{3}-3 C_{1} C_{2}+C_{3} .
$$

\footnotetext{
${ }^{5}$ In [25], there is also an alternative representation of the LLM solutions in terms of the Young diagrams. In that case, $A$ denotes the area of the Young diagram.
} 
In the previous section, we have established the KK maps which relate the above 11dimensional KK modes to the corresponding canonical 4-dimensional gravity fields. These maps are given in (2.45) and (2.54). Using these maps, we can write the asymptotically $\mathrm{AdS}_{4}$ solution to the 4-dimensional gravity equations (2.44) and (2.53) from the KK reduction of the LLM solution. The results are

$$
\begin{aligned}
H_{i j} & =\left[-\frac{\left(L \mu_{0}\right)^{2}}{180}\left(30+\beta_{3}^{2}\right)+\mathcal{O}\left(\mu_{0}^{4}\right)\right] \eta_{i j}, & H_{z z} & =-\frac{\left(L \mu_{0}\right)^{2}}{1440}\left(960+29 \beta_{3}^{2}\right)+\mathcal{O}\left(\mu_{0}^{4}\right) \\
\Psi & =-24 \beta_{3} \mu_{0} z+\mathcal{O}\left(\mu_{0}^{3}\right), & T & =16 \sqrt{3} \mu_{0} z+\mathcal{O}\left(\mu_{0}^{3}\right) .
\end{aligned}
$$

\subsection{One-point function for the CPO with $\Delta=1$}

The vev of a CPO with conformal dimension one in mABJM was obtained in [26, 27]. For clarity of presentation, we shortly review that result here. The CPO in ABJM theory with conformal dimension one, which preserves the $\mathrm{SU}(2) \times \mathrm{SU}(2) \times \mathrm{U}(1)$ global symmetry and has non-vanishing vev, is given by

$$
\mathcal{O}^{(1)}=\frac{1}{2 \sqrt{2}} \operatorname{Tr}\left(Z^{a} Z_{a}^{\dagger}-W^{\dagger a} W_{a}\right) .
$$

Since the supersymmetry of the ABJM theory protects the scalar fields from quantum corrections and the contributions from the multi-trace terms are suppressed by $1 / N$ as compared to single-trace terms, the vevs of the CPOs are exactly determined by the classical values for scalar fields in the large $N$ limit $[26,27]$. Based on this argument, we obtained the vev of the CPO with conformal dimension one for all supersymmetric vacua in large $N$ limit,

$$
\left\langle\mathcal{O}^{(1)}\right\rangle_{m}=\frac{k \mu}{4 \sqrt{2} \pi} \sum_{n=0}^{\infty} n(n+1)\left(N_{n}-N_{n}^{\prime}\right),
$$

where $\langle\cdots\rangle_{m}$ represents the $v e v$ in the mABJM theory and $\mu$ is the mass parameter related to the LLM geometry mass parameter as $\mu=4 \mu_{0}$.

In the gauge/gravity duality, the relation between the conformal dimension of gauge invariant operators and the mass of the dual scalar modes in the 4-dimensional gravity theory is given by

$$
\frac{m_{\phi}^{2} L^{2}}{4}=\Delta(\Delta-3)
$$

In our case the gravity mode dual to the CPO with conformal dimension one is the mode $\Psi$ in (2.44). The gauge/gravity duality dictionary states that, the vev of a CPO with conformal dimension $\Delta$ is determined by the coefficient of $z^{\Delta}$ in the asymptotic expansion of the dual scalar field. According to this rule, the vev of the CPO of conformal dimension one is determined by the asymptotic expansion of $\Psi$ in (3.10) as

$$
\left\langle\mathcal{O}^{(1)}\right\rangle_{m}=-\frac{24 N^{2}}{\sqrt{\lambda}} \mathbb{N} \beta_{3} \mu_{0},
$$


where $\mathbb{N}$ depends on the normaliation of the scalar field $\Psi$ and $\lambda=N / k$ is the 't Hooft coupling constant in the ABJM theory. The overall factor $N^{2} / \sqrt{\lambda}$ is originated from the gauge/gravity dual relation in 4-dimensional gravity,

$$
\frac{1}{16 \pi G_{N}^{(4)}} \sim \frac{N^{2}}{\sqrt{\lambda} L^{2}}
$$

In order to fix the normalization $\mathbb{N}$, we use the identity (see $[26,27]$ for the proof)

$$
\beta_{3}=\frac{3}{A^{\frac{3}{2}}} \sum_{n=0}^{N_{B}} n(n+1)\left(l_{n}-l_{n}^{\prime}\right) .
$$

For $k=1$ and the general $N_{B}$ or the general $k$ and $N_{B}=1$ cases, this identity is valid for any $N \geq 2$. However, for both $k$ and $N_{B}$ greater than one, the right-hand side of (3.16) is only the leading order term in the $\frac{1}{N}$-expansion of $\beta_{3}$. In the large $N$ limit, we note that the leading contribution of $A$ is $A=k N$. Therefore, recalling that the field theory result in (3.12) is obtained in the large $N$ limit, we can fix the normalization as $\mathbb{N}=-\frac{\sqrt{2}}{144 \pi}$ by comparing the field theory result and the gravity result (3.14) in the large $N$ limit. The one-to-one map $\left\{l_{n}, l_{n}^{\prime}\right\} \Longleftrightarrow\left\{N_{n}, N_{n}^{\prime}\right\}$ is also used. Then the vev can be written as

$$
\left\langle\mathcal{O}^{(1)}\right\rangle_{m}=\frac{N^{2} \mu_{0}}{3 \sqrt{2} \pi \sqrt{\lambda}} \beta_{3} .
$$

In $[26,27]$, we have verified that $\beta_{3}$ is independent of $N$, so that the overall normalization factor in (3.17) is proportional to $N^{\frac{3}{2}}$ in the case $k=1$, which is the well-known relation in M2-brane theory. The above relation (3.17) gives an exact dual relation in large $N$ limit for all supersymmetric vacua in mABJM theory and the corresponding LLM geometries with $\mathbb{Z}_{k}$ orbifold.

\subsection{HEE from LLM geometries in 4-dimensions}

According to the RT conjecture, the HEE with a subspace $A$ at a fixed time on the boundary of $(d+1)$-dimensional AdS geometry is given by

$$
S_{A}=\frac{\operatorname{Min}\left(\gamma_{A}\right)}{4 G_{N}}
$$

where $G_{N}$ is the Newton constant in the $(d+1)$-dimensional gravity theory and $\gamma_{A}$ is an area of the surface stretched to the bulk direction, which has the same boundary with the subsystem $A$. The surface is expressed by the induced metric,

$$
g_{i j}^{(0)}=\frac{\partial w^{\mu}}{\partial \sigma^{i}} \frac{\partial w^{\nu}}{\partial \sigma^{j}} g_{\mu \nu}
$$

where $\sigma^{1}, \cdots, \sigma^{d-1}$ are coordinates on the surface, $g_{\mu \nu}$ is the asymptotically $\operatorname{AdS}_{d+1}$ bulk metric, and $w^{0}, w^{1}, \cdots, w^{d}$ are the bulk coordinates. The area $\gamma_{A}$ is given by

$$
\gamma_{A}=\int d^{d-1} \sigma \sqrt{\operatorname{det} \tilde{g}_{i j}}
$$


In section 2, we have constructed a 4-dimensional gravity theory using KK reduction from the 11-dimensional gravity. We showed that, up to the quadratic order in the mass parameter $\mu_{0}$, there are only two scalar fields that are coupled to the 4-dimensional metric. These two scalars carry the information of the asymptotic expansion of the LLM geometry. In the absence of these scalar fields, the geometry is pure $\mathrm{AdS}_{4}$, and the scalar fields induce the deviation from the $\mathrm{AdS}_{4}$ space. In this section, we use the asymptotically $\mathrm{AdS}_{4}$ metric in subsection 3.2 to compute the variation of the $\operatorname{HEE} \delta S_{A}$ from its original value $S_{A}^{0}$ in pure $\mathrm{AdS}_{4}$ geometry.

\subsubsection{HEE from pure AdS geometry in 4-dimensions}

Before we proceed to the calculation of $\delta S_{A}$, let us summarize the calculation of the HEE for the pure $\mathrm{AdS}_{4}$ [2]. The 4-dimensional AdS metric is given by

$$
d s_{\text {AdS }}^{2}=\frac{L_{\text {AdS }}^{2}}{z^{2}}\left(-d t^{2}+d w_{1}^{2}+d w_{2}^{2}+d z^{2}\right),
$$

where $L_{\text {AdS }}$ denotes the radius of the $\mathrm{AdS}_{4}$ geometry and $z$ represents the holographic direction. To obtain the HEE for a subspace $A$ which is a disk of radius $l$, we consider a mapping for the codimension 2 coordinates $\sigma^{1,2}$,

$$
t=\mathrm{constant}, \quad w_{1}=\sigma^{2} \cos \sigma^{1}, \quad w_{2}=\sigma^{2} \sin \sigma^{1}, \quad z=z\left(\sigma^{2}\right) .
$$

The components of the induced metric (3.19) are given by

$$
\tilde{g}_{11}^{(0)}=\frac{L_{\mathrm{AdS}}^{2} \rho^{2}}{z^{2}}, \quad \tilde{g}_{12}^{(0)}=0, \quad \tilde{g}_{22}^{(0)}=\frac{L_{\mathrm{AdS}}^{2}}{z^{2}}\left(1+z^{\prime 2}\right)
$$

where we set $\sigma^{2}=\rho$ and $z^{\prime} \equiv(\partial z / \partial \rho)$. Then the area of the surface $\gamma_{A}$ is given by

$$
\gamma_{A}=\int_{0}^{2 \pi} d \sigma^{1} \int_{0}^{l} d \rho \sqrt{\operatorname{det} \tilde{g}_{i j}}=2 \pi L_{\text {AdS }}^{2} \int_{0}^{l} d \rho \frac{\rho}{z^{2}} \sqrt{1+z^{\prime 2}} .
$$

The solution of $z(\rho)$ which minimizes $\gamma_{A}$ is

$$
z(\rho)=\sqrt{l^{2}-\rho^{2}}
$$

with boundary conditions

$$
z(l)=0, \quad z^{\prime}(0)=0
$$

Computing the minimum area of $\gamma_{A}$, we obtain the HEE,

$$
S_{A}^{(0)}=\frac{\pi L^{2}}{8 G_{N}^{(4)}}\left(\frac{l}{\epsilon}-1\right)
$$

where $\epsilon$ is the UV cut-off in the $z$-direction. 


\subsection{Variation of HEE from LLM geometries in 4-dimensions}

The asymptotically $\mathrm{AdS}_{4}$ metric can be split into the pure AdS part and fluctuations as

$$
\hat{g}_{\mu \nu}=g_{\mu \nu}+H_{\mu \nu}
$$

where $g_{\mu \nu}$ is metric of the pure $\mathrm{AdS}_{4}$. Then the induced metric (3.19) is given by

$$
\tilde{g}_{i j}=\frac{\partial w^{\mu}}{\partial \sigma^{i}} \frac{\partial w^{\nu}}{\partial \sigma^{j}} \hat{g}_{\mu \nu}=\frac{\partial w^{\mu}}{\partial \sigma^{i}} \frac{\partial w^{\nu}}{\partial \sigma^{j}}\left(g_{\mu \nu}+H_{\mu \nu}\right)=\tilde{g}_{i j}^{(0)}+\tilde{H}_{i j},
$$

where $\tilde{g}_{i j}^{(0)}$ is the induced $\mathrm{AdS}_{4}$ metric and $\tilde{H}_{i j}$ is determined from the asymptotic $\mathrm{AdS}_{4}$ solution given in (3.10). Specifically,

$$
\begin{aligned}
& \tilde{H}_{11}=\rho^{2} H_{11}=-\frac{\left(L \mu_{0}\right)^{2} \rho^{2}}{180}\left(30+\beta_{3}^{2}\right), \\
& \tilde{H}_{22}=H_{11}+z^{\prime 2} H_{z z}=-\frac{\left(L \mu_{0}\right)^{2}}{1440}\left[240+8 \beta_{3}^{2}+\left(960+29 \beta_{3}^{2}\right) z^{\prime 2}\right] .
\end{aligned}
$$

Apply the mapping (3.22) to the induced metric (3.29), we obtain the variation of the area,

$$
\delta \gamma_{A}=\frac{1}{2} \int d^{2} \sigma \sqrt{\operatorname{det} \tilde{g}^{(0)}} \tilde{g}^{(0) i j} \tilde{H}_{i j}=\pi \int_{0}^{l} d \rho \sqrt{\operatorname{det} \tilde{g}^{(0)}} \tilde{g}^{(0) i j} \tilde{H}_{i j}
$$

Inserting (3.30) into (3.31), we obtain,

$$
\begin{aligned}
\delta \gamma_{A} & =-\frac{\pi L^{2} \mu_{0}^{2}}{1440} \int_{0}^{l} d \rho \frac{\rho}{\sqrt{1+z^{\prime 2}}}\left[\left(1200+37 \beta_{3}^{2}\right) z^{\prime 2}+16\left(30+\beta_{3}^{2}\right)\right] \\
& =-\frac{\pi L^{2}\left(\mu_{0} l\right)^{2}}{48}\left(32+\beta_{3}^{2}\right) .
\end{aligned}
$$

In the last step of (3.32), we have used the solution of $z(\rho)$ given in (3.25). Therefore, the HEE up to $\mu_{0}^{2}$-order is given by

$$
\begin{aligned}
S_{A}=S_{A}^{(0)}+\delta S_{A} & =\frac{\pi L^{2}}{8 G_{N}^{(4)}}\left[\frac{l}{\epsilon}-1-\frac{4}{3}\left(1+\frac{\beta_{3}^{2}}{32}\right)\left(\mu_{0} l\right)^{2}\right] \\
& =\frac{\sqrt{2} \pi N^{2}}{3 \sqrt{\lambda}}\left[\frac{l}{\epsilon}-1-\frac{4}{3}\left(1+\frac{\beta_{3}^{2}}{32}\right)\left(\mu_{0} l\right)^{2}\right],
\end{aligned}
$$

where the KK reduction relates the 4-dimensional and the 11-dimensional Newton's constant as

$$
\frac{1}{G_{N}^{(4)}}=\frac{\operatorname{vol}\left(S^{7} / \mathbb{Z}_{k}\right)}{G_{N}^{(11)}}=\frac{\pi^{4} L^{7}}{3 k G_{N}^{(11)}}=\frac{8 \sqrt{2} N^{2}}{3 \sqrt{\lambda} L^{2}} .
$$

Here we used the gauge/gravity dual relation in the ABJM theory and the 11-dimensional supergravity, $16 \pi G_{N}^{(11)}=(2 \pi)^{8} l_{\mathrm{P}}^{9}$ and $L^{6}=32 \pi^{2} k N l_{\mathrm{P}}^{6}$. Inserting this into (3.33), we reproduce the HEE from the LLM geometries in 11-dimensions [46]. 
As shown in section 2, the matter fields in the 4-dimensional gravity theory are determined by the 11-dimensional geometry. The equality of the HEE obtained from pure geometrical 11-dimensional gravity theory and the one obtained from the 4-dimensional matter-gravity theory shows that the information of the LLM geometry in the asymptotic limit is exactly encoded in the solutions of the matter fields in 4-dimensions. Reversing this statement, the RT formula may play some role in the construction of geometrical solutions in higher dimensional theories from the solutions of matter fields in lower dimensional theories. It is intriguing to examine this possibility in our setup. However, it is highly non-trivial to achieve this goal because one has to extract the information of metric, which is local, from the HEE, which is non-local.

\subsection{HEE from holographic mapping of vev and source}

In $[26,27]$, we have discussed the gauge/gravity maps between gauge invariant operators in ABJM theory and scalar modes in 11-dimensional supergravity, which encode the information of LLM geometries with $S^{7} / \mathbb{Z}_{k}$ orbifold. Among the five infinite KK towers of scalar modes listed in $[26,27]$, only the two scalar modes in $(2.45)$ are nonvanishing in the leading order of the asymptotic expansion of the LLM geometries. Using the relations between mass and conformal dimension listed in [26, 27], we observe that the scalar field $\Psi$ with $M_{\Psi}^{2}=\left.\frac{I(I-6)}{L^{2}}\right|_{I=2}$ is dual to CPO of conformal dimension $\Delta=\left.\frac{I}{2}\right|_{I=2}=1$ while the pseudoscalar field $T$ with $M_{T}^{2}=\left.\frac{(I-3)(I+3)}{L^{2}}\right|_{I=1}$ is dual to gauge invariant operator with conformal dimension $\Delta=\left.\frac{I+3}{2}\right|_{I=1}=2$. Then, the gauge/gravity dictionary implies ${ }^{6}$ the expansion of these two fields in powers of the holographic coordinate $z$ should read

$$
\Psi(z)=V_{\Psi} z+S_{\Psi} z^{2}+\cdots, \quad T(z)=S_{T} z+V_{T} z^{2}+\cdots,
$$

where the coefficients $V_{\Psi, T}$ are determined by the vevs of the dual operators and the coefficients $S_{\Psi, T}$ are determined by the values of the external sources which are coupled to those operators. Comparing (3.35) with our results in (3.10), we see that $S_{\Psi}=V_{T}=0$, while $V_{\Psi}=-24 \beta_{3} \mu_{0}$ and $S_{T}=16 \sqrt{3} \mu_{0}$. Therefore, one can identity the coefficients of $z$ in $\Psi(z)$ as vevs of the CPO with $\Delta=1$ and the coefficients of $z$ in $T(z)$ as the source of a gauge invariant operator with $\Delta=2$ in 3-dimensions. The coupling of these two scalars to gravity in 4-dimensions causes the deformation of the induced metric in (3.30). More precisely, the $\beta_{3}$ terms in (3.30) are due to the coupling with $\Psi$, while the numerical terms are due to coupling with $T$. As we discussed in the previous subsection, the matric deformation determines the variation of the HEE $\left(\delta S_{A}\right)$, which means that the $\beta_{3}$-term in $\delta S_{A}$ in (3.33) is originated from the vevs of the $\mathrm{CPO},\left\langle\mathcal{O}^{(1)}\right\rangle_{m}$, while the numerical term in $\delta S_{A}$ is originated from the source of the gauge invariant operator $J_{\tilde{\mathcal{O}}^{(2)}}$. The variation

\footnotetext{
${ }^{6}$ The gauge/gravity duality dictionary for the scalar mode is following: under the asymptotic expansion of a scalar field,

$$
\phi\left(z, x_{i}\right)=\phi_{1}\left(x_{i}\right) z+\cdots+\phi_{d-\Delta}\left(x_{i}\right) z^{d-\Delta}+\cdots+\phi_{\Delta}\left(x_{i}\right) z^{\Delta}+\cdots,
$$

the vev of the dual gauge invariant operator with conformal dimension $\Delta$ is proportional to $\phi_{\Delta}\left(x_{i}\right)$ and the source of that operator is proportional to $\phi_{d-\Delta}\left(x_{i}\right)$.
} 
of HEE is actually related to the squares of the vevs and the source term. Therefore, up to $\mu_{0}^{2}$-order in the large $N$ limit, the HEE can be written as

$$
\delta S_{A}=-\frac{4 \sqrt{2} \pi N^{2} l^{2}}{\sqrt{\lambda}}\left[\frac{1}{9}\left(J_{\tilde{\mathcal{O}}^{(2)}}\right)^{2}+\frac{1}{16}\left(\frac{\pi \sqrt{\lambda}\left\langle\mathcal{O}^{(1)}\right\rangle_{m}}{N^{2}}\right)^{2}\right],
$$

where we set the source of the operator $\tilde{\mathcal{O}}^{(2)}$ as $J_{\tilde{\mathcal{O}}^{(2)}}=\mu_{0}$.

The CPO of conformal dimension one is as in (3.11), and the gauge invariant operator $\tilde{\mathcal{O}}^{(2)}$ is built from the fermionic fields of the ABJM theory, $\left(\psi_{A}, A=1,2,3,4\right)$, and is given by [53]

$$
\tilde{\mathcal{O}}^{(2)}=\tilde{C}_{A}^{B} \operatorname{Tr}\left(\psi^{\dagger A} \psi_{B}\right)
$$

where $\tilde{C}_{A}^{B}$ are traceless. See [54] for the source of the mass deformation by adding fermion mass terms in the dual field theory. In general the CPOs of conformal dimension $\Delta$ are given by

$$
\mathcal{O}^{(\Delta)}=C_{A_{1}, \cdots, A_{\Delta}}^{B_{1}, \cdots, B_{\Delta}} \operatorname{Tr}\left(Y^{A_{1}} Y_{B_{1}}^{\dagger} \cdots Y^{A_{\Delta}} Y_{B_{\Delta}}^{\dagger}\right)
$$

The gauge invariant operators $\tilde{\mathcal{O}}^{(\Delta)}$ are descendents of the CPOs and they are given by

$$
\tilde{\mathcal{O}}^{(\Delta)}=\tilde{C}_{a, A_{1}, \cdots, A_{\Delta-2}}^{b, B_{1}, \cdots, B_{\Delta-2}} \operatorname{Tr}\left(\psi^{\dagger a} \psi_{b} Y^{A_{1}} Y_{B_{1}}^{\dagger} \cdots Y^{A_{\Delta-2}} Y_{B_{\Delta-2}}^{\dagger}\right) .
$$

The CPOs $\mathcal{O}^{(\Delta)}$ are dual to the scalar KK modes $\Psi^{I}(I=2,4,6, \cdots)$ with $\Delta=\frac{I}{2}$, and the gauge invariant operators $\tilde{\mathcal{O}}^{(\Delta)}$ are dual to the pseudoscalar KK modes $T^{I}(I=1,3,5, \cdots)$ with $\Delta=\frac{I+3}{2}$. We obtain the expansions in holographic coordinate, for those two KK towers of dual scalars from the asymptotic expansion of the LLM solutions, which are given by

$$
\begin{array}{lll}
\Psi^{2+4 i}(z)=\tilde{\psi}_{1}\left(\mu_{0} z\right)+\tilde{\psi}_{3}\left(\mu_{0} z\right)^{3}+\cdots+\tilde{\psi}_{\Delta}\left(\mu_{0} z\right)^{\Delta}+\tilde{\psi}_{\Delta+2}\left(\mu_{0} z\right)^{\Delta+2}+\cdots, & (\Delta=1+2 i), \\
\Psi^{4+4 i}(z)=\tilde{\psi}_{2}\left(\mu_{0} z\right)^{2}+\tilde{\psi}_{4}\left(\mu_{0} z\right)^{4}+\cdots+\tilde{\psi}_{\Delta}\left(\mu_{0} z\right)^{\Delta}+\tilde{\psi}_{\Delta+2}\left(\mu_{0} z\right)^{\Delta+2}+\cdots, & (\Delta=2+2 i), \\
T^{1+4 i}(z)=t_{1}\left(\mu_{0} z\right)+t_{3}\left(\mu_{0} z\right)^{3}+\cdots+t_{\Delta-1}\left(\mu_{0} z\right)^{\Delta-1}+t_{\Delta+1}\left(\mu_{0} z\right)^{\Delta+1}+\cdots, & (\Delta=2+2 i), \\
T^{3+4 i}(z)=t_{2}\left(\mu_{0} z\right)^{2}+t_{4}\left(\mu_{0} z\right)^{4}+\cdots+t_{\Delta-1}\left(\mu_{0} z\right)^{\Delta-1}+t_{\Delta+1}\left(\mu_{0} z\right)^{\Delta+1}+\cdots, & (\Delta=3+2 i),
\end{array}
$$

where $i=0,1,2, \cdots$. As mentioned before, in these expansions the coefficient of $z^{\Delta}$ is determined by the vev of the dual operator while the coefficient of $\left.z^{d-\Delta}\right|_{d=3}=z^{3-\Delta}$ is determined by the value of the external source which is coupled to the operator. Among the towers of the KK modes in (3.40), the only mode which has nonvanishing coefficient of $z^{3-\Delta}$ is $T=\left.T^{1+4 i}\right|_{i=0}$. Therefore, the dual operator $\tilde{\mathcal{O}}^{(2)}$ is the only operator which is coupled to an external source, while the rest have no external source term. In addition, from (3.40), we see that all the gauge invariant operators dual to the pseudo-scalar fields $T^{I}$ have vanishing vevs whereas all the CPOs which are dual to the scalar fields $\Psi^{I}$ have non-vanishing vevs. This suggests that, the variation of the HEE $\left(\delta S_{A}\right)$ can depend on the source term of the 
gauge invariant operator $\tilde{\mathcal{O}}^{(2)}$ and the vevs of the $\operatorname{CPOs} \mathcal{O}^{(\Delta)}(\Delta=1,2,3 \cdots)$. However, as can be seen from (3.40), for $\Delta \geq 2$, the vevs are at least quadratic in $\mu_{0}$, and cannot contribute to $\delta S_{A}$ at quadratic order in $\mu_{0}$. Therefore, at quadratic order in $\mu_{0}$, the variation of HEE is fully determined by the source term of the operator $\tilde{\mathcal{O}}^{(2)}$ and the vev of the $\mathrm{CPO} \mathcal{O}^{(1)}$, which is the result in (3.36).

\subsection{Comments on relative entropy and Fisher information}

The variation of the EE, $\delta S_{A}$ in (3.33), is connected with the relative entropy, which is defined as

$$
S(\rho \| \sigma) \equiv \operatorname{tr}(\rho \log \rho)-\operatorname{tr}(\rho \log \sigma)
$$

where $\rho$ is a deformed density matrix from some reference density matrix $\sigma$. For the ball-shaped region $A$, the relative entropy is represented as

$$
S\left(\rho_{A} \| \sigma_{A}\right)=\Delta\left\langle H_{A}\right\rangle-\Delta S_{A}
$$

where $H_{A}$ is the modular Hamiltonian associated with the region $A$. See below for details. At first order in the deformation parameter, the relative entropy vanishes and (3.42) becomes the first law of the EE. Therefore, the leading nonvanishing contribution to relative entropy is quadratic in the deformation parameter. In our case the deformation parameter is $\mu_{0}$, and due to the supersymmetry of the mABJM theory, $\Delta\left\langle H_{A}\right\rangle_{\mu_{0}^{2}}=0$ (see the discussion below the equation (4.3)). Then using the result in (3.33), we have

$$
\left.S\left(\rho_{A} \| \sigma_{A}\right)\right|_{\mu_{0}^{2}}=\frac{\pi L^{2}}{6 G_{N}^{(4)}}\left(1+\frac{\beta_{3}^{2}}{32}\right)\left(\mu_{0} l\right)^{2} .
$$

The relative entropy is positive definite as a measure of distance between two quantum states and monotonically increasing with the size of the subsystem [12, 42, 43, 55-57]. The relation in (3.43) represents those properties clearly. The positivity of the relative entropy states the positivity of the Fisher information metric with one deformation parameter,

$$
F_{\mu_{0} \mu_{0}}=\frac{d^{2}}{d \mu_{0}^{2}} S\left(\rho_{A} \| \sigma_{A}\right)=\frac{\pi L^{2} l^{2}}{3 G_{N}^{(4)}}\left(1+\frac{\beta_{3}^{2}}{32}\right) .
$$

This quantity is also known as the fidelity susceptibility. In [43], it was shown that the Fisher information of the reduced density matrix of a ball-shaped subregion at the CFT vacuum is connected to the canonical energy [58] for perturbations in the corresponding Rindler wedge of the AdS geometry. See also [59] for the canonical energy by using the Euler-Lagrange expression and its connection to the Fisher information. In most cases, for instance [38, 41-43, 60], the second order deformation in the gravity is connected to nonvanishing vev of gauge invariant operator in QFT. Though the field fluctuations in gravity side in our case are originated from the vev and source of gauge invariant operators, we expect that the interpretation of the Fisher information in QFT as the canonical energy in the gravity side is correct since the roles of the dual fields $\Psi$ and $T$ in gravity side are indistinguishable. 


\section{Gravity from entanglement and RG flow}

In the previous section, we have determined the HEE by applying the RT formula in 4dimensional gravity, which is obtained from the KK reduction of the 11-dimensional gravity on the LLM geometry. At the quadratic order in $\mu_{0}$, our result is in complete agreement with the one obtained by applying the RT formula in the 11-dimensional supergravity before the KK reduction [46]. This indirectly proves that the solution of the 4-dimensional gravity theory we have built contains all the information of the 11-dimensional LLM geometry near the UV fixed point. In the dual gauge theory, the asymptotic limit of the LLM geometry describes the RG flow from the UV fixed point where the ABJM theory lives. In this section we discuss the first law-like relation for the EE when there is RG flow due to relevant perturbations from the UV fixed point.

\subsection{Emergent gravity from relevant perturbations in CFT}

We consider an Euclidean CFT action with a relevant deformation,

$$
I=I^{(0)}+\tilde{\lambda} \int d^{d} w \mathcal{O}^{(\Delta)},
$$

where $I^{(0)}$ is the $d$-dimensional CFT action, $\mathcal{O}^{(\Delta)}$ is a gauge invariant operator with conformal dimension $\Delta$, and $\tilde{\lambda}$ is the deformation parameter with mass dimension $d-\Delta>0$. In QFT, the EE is defined as

$$
S_{A}=-\operatorname{tr}\left(\rho_{A} \ln \rho_{A}\right),
$$

where the total space of states is divided into two subregions $A, B$ and $\rho_{A}$ is the reduced density matrix of the subregion $A$ at a given time. Here we consider the subregion $A$ is in a shape of $(d-1)$-dimensional ball of radius $l$. Under the relevant deformation (4.1), the density matrix is deformed as $\rho_{A}=\rho_{A}^{(0)}+\delta \rho_{A}$ with the matrix $\rho_{A}^{(0)}$ is for the undeformed CFT.

The EE is calculated in the path integral formalism using the perturbative expansion in the deformation parameter $\tilde{\lambda}$. At the linear order in $\tilde{\lambda}$, the perturbative evaluation of the EE produces the relation $\delta S=\delta\left\langle H_{A}\right\rangle_{\tilde{\lambda}}$, where $H_{A}$ is the modular Hamiltonian defined as $H_{A} \equiv-\ln \rho_{A}$. This relation is known as the first law of the EE [10,12]. For a ball-shaped subregion $A$, the modular Hamiltonian is expressed in terms of energy-momentum tensor (see below). At the quadratic order of the perturbative expansion, it was pointed out that the variation of EE gets contributions from the two point function $\left\langle\mathcal{O}^{(\Delta)} \mathcal{O}^{(\Delta)}\right\rangle$ and the three point function $\left\langle H_{A} \mathcal{O}^{(\Delta)} \mathcal{O}^{(\Delta)}\right\rangle[33,34]$. Later, it was found that in the evaluation of the two point function $\left\langle\mathcal{O}^{(\Delta)} \mathcal{O}^{(\Delta)}\right\rangle$ there exists an additional finite contribution at the $\tilde{\lambda}^{2}$-order, which comes from the non-commutative property between the matrix representations of $\rho_{A}^{(0)}$ and $\delta \rho_{A}$ [32]. As we will see later, this finite term reflects the deviation of EE away from the UV fixed point under the relevant deformation and was identified quantitatively in the holographic picture via the RT conjecture.

Next, we follow [32] and briefly discuss the two terms contributing to the variation of $\mathrm{EE}$ at $\tilde{\lambda}^{2}$-order. The first one is given by

$$
\delta S^{(1)}=\frac{\tilde{\lambda}^{2}}{2} \int d^{d} w \int d^{d} w^{\prime}\left\langle H_{A} \mathcal{O}^{(\Delta)}(w) \mathcal{O}^{(\Delta)}\left(w^{\prime}\right)\right\rangle_{0}=\delta\left\langle H_{A}\right\rangle_{\tilde{\lambda}^{2}},
$$


where $\langle\cdots\rangle_{0}$ denotes the $n$-point functions of the undeformed theory. The result in (4.3) has also been obtained in [33, 34]. For the ball-shaped region $A$, the explicit form of the modular Hamiltonian $H_{A}$ for the $d$-dimensional CFT is obtained in [61],

$$
H_{A}=2 \pi \int_{B\left(l, \vec{w}_{0}\right)} d \Sigma^{i} \zeta^{j} T_{i j}=2 \pi \int_{B\left(l, \vec{w}_{0}\right)} d^{d-1} w \frac{l^{2}-\left|\vec{w}-\vec{w}_{0}\right|^{2}}{2 l} T_{t t}\left(t_{0}, \vec{w}\right),
$$

where $i=0,1, \cdots, d-1$, the ball $B\left(l, \vec{w}_{0}\right)$ is on a time slice $t=t_{0}$, it is of radius $l$ and centered at $\vec{w}=\vec{w}_{0}$. The $d \Sigma^{i}$ is the volume form on the $(d-1)$-dimensional surface perpendicular to a unit vector in $i$-th direction and $\zeta$ is the conformal Killing vector defined as

$$
\zeta=\left(\frac{l^{2}-\rho^{2}-t^{2}}{2 l}\right) \partial_{t}-\frac{\rho t}{l} \partial_{\rho}
$$

with radius $\rho=\sqrt{w_{1}^{2}+\cdots+w_{d-1}^{2}}$. Here $T_{t t}$ denotes the $(t t)$-component of the energymomentum tensor in $d$-dimensional CFT. Inserting (4.4) into (4.3), the calculation of $\delta S^{(1)}$ is reduced to the evaluation of the three point function $\left\langle T_{t t}(w) \mathcal{O}^{(\Delta)}\left(w^{\prime}\right) \mathcal{O}^{(\Delta)}\left(w^{\prime \prime}\right)\right\rangle_{0}$. In the mABJM theory with supersymmetric discrete Higgs vacua, the three point function is vanishing in the large $N$ limit. In order to see this, we expand the field near the vacua as $Y^{A=1,2,3,4}=Y_{0}^{A}+\tilde{Y}^{A}$ with the vacuum configuration $Y_{0}^{A}$. Then the gauge invariant operators are written as

$$
\mathcal{O}^{(\Delta)}(w)=\mathcal{O}_{0}^{(\Delta)}+\sum_{i} \tilde{\mathcal{O}}_{i}^{(\Delta)}(w)
$$

where $\left.\mathcal{O}_{0}^{(\Delta)} \equiv \mathcal{O}^{(\Delta)}(w)\right|_{Y^{A}=Y_{0}^{A}}$ and $\tilde{\mathcal{O}}_{i}^{(\Delta)}$ 's are operators expanded around the vacuum $Y_{0}^{A}$. Then the three point function can formally be written as

$$
\begin{aligned}
\left\langle T_{t t}(w) \mathcal{O}^{(\Delta)}\left(w^{\prime}\right) \mathcal{O}^{(\Delta)}\left(w^{\prime \prime}\right)\right\rangle_{0}= & \sum_{i, j}\left\langle T_{t t}(w) \tilde{\mathcal{O}}_{i}^{(\Delta)}\left(w^{\prime}\right) \tilde{\mathcal{O}}_{j}^{(\Delta)}\left(w^{\prime \prime}\right)\right\rangle_{0}+\left(\mathcal{O}_{0}^{(\Delta)}\right)^{2}\left\langle T_{t t}(w)\right\rangle_{0} \\
& +\mathcal{O}_{0}^{(\Delta)} \sum_{i}\left[\left\langle T_{t t}(w) \tilde{\mathcal{O}}_{i}^{(\Delta)}\left(w^{\prime}\right)\right\rangle_{0}+\left\langle T_{t t}(w) \tilde{\mathcal{O}}_{i}^{(\Delta)}\left(w^{\prime \prime}\right)\right\rangle_{0}\right] .
\end{aligned}
$$

In CFT, the conformal invariance dictates that the two point function $\left\langle\mathcal{O} T_{\mu \nu}\right\rangle_{0}$ is vanishing. In addition, the first term on the right-hand side in the above equation is a multi-trace term and is suppressed by $1 / N$ as compared with the single trace terms. Therefore the three point function in the large $N$ limit is given by

$$
\left\langle T_{t t}(w) \mathcal{O}^{(\Delta)}\left(w^{\prime}\right) \mathcal{O}^{(\Delta)}\left(w^{\prime \prime}\right)\right\rangle_{0}=\left(\mathcal{O}_{0}^{(\Delta)}\right)^{2}\left\langle T_{t t}(w)\right\rangle_{0}+\frac{1}{N}-\text { corrections }
$$

Since the mABJM theory is a supersymmetric gauge theory, the vev of the energymomentum tensor $\left\langle T_{t t}(w)\right\rangle_{0}$ is vanishing. Therefore, we expect that $\delta S^{(1)}$ for the mABJM theory is vanishing in the large $N$-limit.

The second contribution to the variation of the EE is obtained in [32] and it is given by

$$
\delta S^{(2)}=-2 \pi \int_{\mathcal{H}^{+}} d \Sigma^{\mu} \xi^{\nu} \tilde{T}_{\mu \nu}
$$


where $\mu, \nu=0,1, \cdots d$ including one additional direction, which will be identified in dual gravity theory as the holographic direction. Here the integration is performed over the region $\mathcal{H}^{+}$, which is the future part of the Rindler horizon in the emergent $\operatorname{AdS}_{d+1}$ space and $d \Sigma^{\mu}$ is the surface element on the horizon. The energy-momentum tensor $\tilde{T}_{\mu \nu}$ of an auxiliary scalar field $\tilde{\phi}$, is

$$
\tilde{T}_{\mu \nu}=\nabla_{\mu} \tilde{\phi} \nabla_{\nu} \tilde{\phi}-\frac{1}{2} g_{\mu \nu}^{(0)}\left(\nabla_{\lambda} \phi \nabla^{\lambda} \tilde{\phi}+m^{2} \tilde{\phi}^{2}\right)
$$

which satisfies the conservation law $\nabla_{\mu} \tilde{T}^{\mu \nu}=0$, and this means the $\delta S^{(2)}$ in (4.9) is a conserved charge. The field $\tilde{\phi}$ satisfies the Klein-Gordon equation,

$$
\nabla^{2} \tilde{\phi}-\frac{\Delta(\Delta-d)}{L_{\mathrm{AdS}}^{2}} \tilde{\phi}=0
$$

with some boundary conditions at $z=0$. For calculation of $\delta S^{(2)}$, we consider an explicit example of the emergent space. To do that, we introduce the $\operatorname{AdS}_{d+1}$ geometry in the Poincare patch,

$$
d s^{2}=\frac{1}{z^{2}}\left(-d t^{2}+d z^{2}+d \rho^{2}+\rho^{2} d \Omega_{d-2}^{2}\right),
$$

where $d \Omega_{d-2}^{2}$ is the line element on $S^{d-2}$ with unit radius. The Killing vector $\zeta$ in (4.5) of the $d$-dimensional CFT extends to a Killing vector in the emergent bulk geometry,

$$
\xi=\frac{l^{2}-z^{2}-\rho^{2}-t^{2}}{2 l} \partial_{t}-\frac{t}{l}\left(z \partial_{z}+\rho \partial_{\rho}\right)
$$

When we choose a spatial slice at $t=0$, the AdS Rindler horizon is reduced to the minimal surface satisfying the relation $z(\rho)=\sqrt{l^{2}-\rho^{2}}$ in (3.25). Then the variation $\delta S^{(2)}$ in (4.9) is given by

$$
\delta S^{(2)}=-\frac{\pi \Omega_{d-2} L_{\text {AdS }}^{d-1}}{l} \int_{z_{\Lambda}}^{l} d z z^{1-d} \int_{0}^{\sqrt{l^{2}-z^{2}}} d \rho \rho^{d-2}\left(l^{2}-z^{2}-\rho^{2}\right) \tilde{T}_{t t},
$$

where $\Omega_{d-2}=\frac{2 \pi^{(d-1) / 2}}{\Gamma((d-1) / 2)}$. We used the volume form $d \Sigma^{t}=L_{\text {AdS }}^{d-1} z^{1-d} d z \rho^{d-2} d \rho d \Omega_{d-2}$ and the Killing vector $\xi^{t}=\frac{l^{2}-z^{2}-\rho^{2}}{2 l}$, and also introduced the cutoff scale $z_{\Lambda}$ to regularize the divergence at $z=0$.

In the $z \rightarrow 0$ limit, the $\tilde{\phi}$ configuration satisfying the equation (4.11) is given by

$$
\tilde{\phi}(z, w) \longrightarrow V_{\tilde{\lambda}}(w) z^{\Delta}+S_{\tilde{\lambda}}(w) z^{d-\Delta} .
$$

Since this auxiliary field is absent in the undeformed theory, the coefficients $V_{\tilde{\lambda}}$ and $S_{\tilde{\lambda}}$ also depend on the deformation parameter. ${ }^{7}$ Inserting the solution (4.15) into (4.14), one can obtain $\delta S^{(2)}$ in the path integral method. For a very small ball-shaped region, $l \ll L_{\mathrm{AdS}}$, the coefficients $V_{\tilde{\lambda}}$ and $S_{\tilde{\lambda}}$ can be regarded as constants, which are consistent with our case. In the literature the $\Delta<\frac{d}{2}$ and $\Delta>\frac{d}{2}$ cases are treated separately, whereas the $\Delta=\frac{d}{2}$ case needs a special treatment [37, 44].

\footnotetext{
${ }^{7}$ If one identifies this auxiliary field with a scalar field in a gravity theory, the gauge/gravity dictionary dictates that $V_{\tilde{\lambda}}$ corresponds to the vev of a gauge invariant operator with conformal dimension $\Delta$, while $S_{\tilde{\lambda}}$ corresponds to the source coupled to the operator.
} 
- $\Delta<\frac{d}{2}$ case. To obtain the leading contribution for the small value of $\tilde{\lambda}$ in $\delta S^{(2)}$, it is enough to consider the asymptotic behavior of $\tilde{\phi}(z, w)$ as

$$
\tilde{\phi}(z, w)=V_{\tilde{\lambda}} z^{\Delta}
$$

where $V_{\tilde{\lambda}}=\mathbb{N}_{V} \tilde{\lambda}$ with a numerical factor $\mathbb{N}_{V}$. Then we obtain the $(t t)$-component of the energy-momentum tensor, $T_{t t}^{A}=V_{\tilde{\lambda}}^{2} z^{2 \Delta-2} \Delta\left(\Delta-\frac{d}{2}\right)$. Inserting this into (4.14), we obtain $[12,37]$

$$
\begin{aligned}
& \delta S^{(2)}=\frac{\pi \Omega_{d-2} \mathbb{N}_{V}^{2} \tilde{\lambda}^{2} L_{\mathrm{AdS}}^{d-1}}{l} \Delta\left(\frac{d}{2}-\Delta\right) \int_{z_{\Lambda}}^{l} z^{2 \Delta-d-1} \int_{0}^{\sqrt{l^{2}-z^{2}}} d \rho \rho^{d-2}\left(l^{2}-z^{2}-\rho^{2}\right) \\
& =-\frac{\pi \Omega_{d-2} \mathbb{N}_{V}^{2} \tilde{\lambda}^{2} L_{\mathrm{AdS}}^{d-1} l^{2 \Delta} \Delta \Gamma\left(\frac{d+3}{2}\right) \Gamma\left(\Delta-\frac{d}{2}+1\right)}{\left(d^{2}-1\right) \Gamma\left(\frac{3}{2}+\Delta\right)}+\delta S_{\mathrm{div}}^{(2)},
\end{aligned}
$$

where $\delta S_{\text {div }}^{(2)}$ includes the divergent pieces depending the UV-cutoff $z_{\Lambda}$,

$$
\delta S_{\mathrm{div}}^{(2)}=-\frac{\pi \Omega_{d-2} \mathbb{N}_{V}^{2} \tilde{\lambda}^{2} L_{\mathrm{AdS}}^{d-1} l^{d} \Delta}{\left(d^{2}-1\right) z_{\Lambda}^{d-2 \Delta}}+\mathcal{O}\left(z_{\Lambda}^{-d+2 \Lambda+2}\right) .
$$

- $\Delta>\frac{d}{2}$ case. In order to extract the leading contribution for the small value of $\tilde{\lambda}$, we consider the asymptotic behavior of $\tilde{\phi}(z, w)$ as

$$
\tilde{\phi}(z, w)=S_{\tilde{\lambda}} z^{d-\Delta}
$$

where $S_{\tilde{\lambda}}=\mathbb{N}_{S} \tilde{\lambda}$ with a numerical factor $\mathbb{N}_{S}$. Then we obtain the $(t t)$-component of the energy-momentum tensor, $T_{t t}^{A}=S_{\tilde{\lambda}}^{2} z^{2 d-2 \Delta-2}\left(\Delta-\frac{d}{2}\right)(\Delta-d)$. Inserting this into (4.14), we obtain the result in [32],

$$
\begin{aligned}
\delta S^{(2)} & =\frac{\pi \Omega_{d-2} \mathbb{N}_{S}^{2} \tilde{\lambda}^{2} L_{\mathrm{AdS}}^{d-1}}{l}(d-\Delta)\left(\Delta-\frac{d}{2}\right) \int_{z_{\Lambda}}^{l} z^{d-2 \Delta-1} \int_{0}^{\sqrt{l^{2}-z^{2}}} d \rho \rho^{d-2}\left(l^{2}-z^{2}-\rho^{2}\right) \\
& =-\frac{\pi \Omega_{d-2} \mathbb{N}_{S}^{2} \tilde{\lambda}^{2} L_{\mathrm{AdS}}^{d-1} l^{2(d-\Delta)}(d-\Delta) \Gamma\left(\frac{d+3}{2}\right) \Gamma\left(\frac{d}{2}-\Delta+1\right)}{\left(d^{2}-1\right) \Gamma\left(\frac{3}{2}+d-\Delta\right)}+\delta S_{\mathrm{div}}^{(2)},
\end{aligned}
$$

where

$$
\delta S_{\mathrm{div}}^{(2)}=-\frac{\pi \Omega_{d-2} \mathbb{N}_{S}^{2} \tilde{\lambda}^{2} L_{\mathrm{AdS}}^{d-1} l^{d}(d-\Delta)}{\left(d^{2}-1\right) z_{\Lambda}^{2 \Delta-d}}+\mathcal{O}\left(z_{\Lambda}^{d-2 \Lambda+2}\right)
$$

The leading finite contribution in (4.20) was also obtained in [40] using the RT formula.

In the calculations of $\delta S^{(1)}$ in (4.3) and $\delta S^{(2)}$ in (4.9), there exist divergent terms. One needs to subtract those terms by adding an appropriate count term in $\tilde{\lambda}^{2}$-order

$$
\delta S_{A}=\left\langle H_{A}\right\rangle_{\tilde{\lambda}}-2 \pi \int_{\mathcal{H}^{+}} d \Sigma^{\mu} \xi^{\nu} \tilde{T}_{\mu \nu}+S_{c t}
$$




\subsection{Gravity from entanglement in the mABJM theory}

In the previous subsection, using the path integral method in $d$-dimensional CFT in the presence of the relevant deformation (4.1), we have summarized that some part of $\delta S$ in $\tilde{\lambda}^{2}$-order becomes a conserved charge in $(d+1)$-dimensional theory. The conserved charge is defined by introducing one additional coordinate $z$ and one auxiliary field $\tilde{\phi}$. The appearance of the additional coordinate indicates the emergence of gravity from the entanglement in QFT. We expect the emergent gravity identifies with the gravity theory which is dual to the QFT. In particular, the energy-momentum tensor of the auxiliary field is expected to be identified with the energy-momentum tensor of a dynamical scalar field in the dual gravity theory.

In this paper, we have constructed a 4-dimensional gravity theory with the matter sector composed of two scalar fields $\Psi$ and $T$. The result we summarized in the previous subsection suggests that these two scalar fields should emerge from the calculation of the variation of the EE in the 3-dimensional dual mABJM theory. In this subsection, we discuss this phenomena in detail and show the emergence of 4-dimensional Einstein equation from the EE analysis.

In order to calculate the quantity $\delta S^{(2)}$ in (4.9) from the energy-momentum tensor defined in (2.48), we treat the contributions from $\Psi$ and $T$ separately. The operator which is dual to the field $\Psi$ is of conformal dimension one and it corresponds to the case $\Delta<\frac{d}{2}$, while that of the field $T$ is of conformal dimension two and corresponds to the case $\Delta>\frac{d}{2}$. Comparing the energy-momentum tensors in (2.48) and (4.10), we need to rescale the scalar fields as $\tilde{\Psi}=\sqrt{A_{\psi}} \Psi$ and $\tilde{T}=\sqrt{A_{t}} T$ and then asymptotic behaviours of the scalar fields in (3.10) are rescaled as

$$
\tilde{\Psi}=-24 \sqrt{A_{\psi}} \beta_{3} \mu_{0} z+\mathcal{O}\left(\mu_{0}^{3}\right), \quad \tilde{T}=16 \sqrt{3} \sqrt{A_{t}} \mu_{0} z+\mathcal{O}\left(\mu_{0}^{3}\right) .
$$

The energy-momentum tensors for $\tilde{\Psi}$ and $\tilde{T}$ up to $\mu_{0}^{2}$-order are read from (4.10),

$$
\begin{array}{ll}
\tilde{T}_{i j}^{(\tilde{\Psi})}=288 A_{\psi} \beta_{3}^{2} \mu_{0}^{2} \eta_{i j}, & \tilde{T}_{z z}^{(\tilde{\Psi})}=864 A_{\psi} \beta_{3}^{2} \mu_{0}^{2}, \\
\tilde{T}_{i j}^{(\tilde{T})}=384 A_{t} \mu_{0}^{2}, & \tilde{T}_{z z}^{(\tilde{T})}=1152 A_{t} \mu_{0}^{2} .
\end{array}
$$

For the field $\tilde{\Psi}$ in (4.23), we obtain the variation of the EE from (4.17) after the cancellation of the divergent term,

$$
\delta S_{\Psi}^{(2)}=-96 \pi^{2} L^{2} A_{\psi} \beta_{3}^{2}\left(\mu_{0} l\right)^{2} .
$$

Similarly, for the scalar field $\tilde{T}$, the result is obtained from (4.20),

$$
\delta S_{T}^{(2)}=-128 \pi^{2} L^{2} A_{t}\left(\mu_{0} l\right)^{2} .
$$

Therefore, the total variation of the EE is given by

$$
\delta S^{(2)}=\delta S_{\Psi}^{(2)}+\delta S_{T}^{(2)}=-128 \pi^{2} L^{2}\left(A_{t}+\frac{3 A_{\psi} \beta_{3}^{2}}{4}\right)\left(\mu_{0} l\right)^{2} .
$$


In the subsection 4.1 , we argued that the quantity $\delta S^{(1)}$ for the mABJM theory is vanishing in the large $N$ limit. This is because of vanishing vacuum energy density $\left\langle T_{i j}(w)\right\rangle_{0}=0$ in supersymmetric theories. Now we examine again the vanishing of $\delta S^{(1)}$ for the mABJM theory using the gauge/gravity duality dictionary. To that end, we start from the deformed 4-dimensional metric in (3.10) and (3.28),

$$
d s^{2}=\frac{L^{2}}{4 z^{2}}\left[\left(1-\mathcal{B}\left(\mu_{0} z\right)^{2}+\mathcal{O}\left(z^{4}\right)\right) d z^{2}+\left(1-\mathcal{A}\left(\mu_{0} z\right)^{2}+\mathcal{O}\left(z^{4}\right)\right) \eta_{i j} d x^{i} d x^{j}\right],
$$

where

$$
\mathcal{A}=\frac{1}{45}\left(30+\beta_{3}^{2}\right), \quad \mathcal{B}=\frac{1}{360}\left(960+29 \beta_{3}^{2}\right) .
$$

The FG coordinate system is convenient to read the vev of the energy-momentum tensor from the asymptotic expansion of the metric. Therefore, we apply the coordinate transformation,

$$
z \longrightarrow \tilde{z}=z-\frac{\mu_{0}^{2} \mathcal{B}}{4} z^{3}
$$

to the metric (4.28) in order to write it in FG-coordinate system,

$$
d s^{2}=\frac{L^{2}}{4 \tilde{z}^{2}}\left[d \tilde{z}^{2}+\left(1-\frac{1}{2}(2 \mathcal{A}+\mathcal{B})\left(\mu_{0} \tilde{z}\right)^{2}+\mathcal{O}\left(\tilde{z}^{4}\right)\right) \eta_{i j} d x^{i} d x^{j}\right] .
$$

This asymptotic behavour of the metric tells us the fact that $\left\langle T_{i j}\right\rangle_{0}$ is vanishing. ${ }^{8}$ This confirms the claim that $\delta S^{(1)}=0$ for the mABJM theory.

In conclusion, the quantity $\delta S^{(2)}$ represents the full variation of the EE in the mABJM theory, which coincides with the variation of area obtained in (3.32). On the other hand, for a general metric perturbation, the variation of the area is also given $[15,66,67]$,

$$
\delta \gamma_{A}=-\int_{\mathcal{H}_{0}} d \Sigma^{\mu} \xi^{\nu} \delta G_{\mu \nu}+\delta \gamma_{A}^{(c t)}
$$

where $\mathcal{H}_{0}$ is a region on the space-like surface $t=0$ squeezed between the minimum area and the disk $A$ with the UV cut-off at $z=z_{\Lambda}$. The term $\delta \gamma_{A}^{(c t)}$ is introduced to cancel out the divergences which arise from $z_{\Lambda} \rightarrow 0$ limit. Here the variation of the Einstein tensor $\delta G_{\mu \nu}$ is read from the left-hand side of (2.51),

$$
\begin{aligned}
\delta G_{\mu \nu}= & \frac{1}{2}\left(-\square H_{\mu \nu}+\nabla^{\rho} \nabla_{\mu} H_{\rho \nu}+\nabla^{\rho} \nabla_{\nu} H_{\rho \mu}-\nabla_{\mu} \nabla_{\nu} H\right)+\frac{12}{L^{2}} H_{\mu \nu}-\frac{6}{L^{2}} g_{\mu \nu} H \\
& -\frac{1}{2} g_{\mu \nu}\left(\nabla^{\rho} \nabla^{\sigma} H_{\rho \sigma}-\square H\right) .
\end{aligned}
$$

${ }^{8}$ For the metric in the FG coordinate system in $(d+1)$-dimensional gravity theory,

$$
d s^{2}=\frac{L_{\mathrm{AdS}}^{2}}{z^{2}}\left(d z^{2}+g_{i j}(x, z) d x^{i} d x^{j}\right),
$$

where $L_{\mathrm{AdS}}$ is the radius of the $\mathrm{AdS}_{d+1}$ geometry and the metric is expanded as $g_{i j}(x, z)=g_{(0) i j}(x)+$ $z^{2} g_{(2) i j}(x)+\cdots+z^{d} g_{(d) i j}(x)+\cdots$ in the asymptotic limit, the vev of the energy-momentum tensor operator is given by [62-65]

$$
\left\langle T_{i j}\right\rangle=\frac{d L_{\mathrm{AdS}}^{d-1}}{16 \pi G_{N}} g_{(d) i j}
$$


Inserting (3.10) into (4.35), we obtain

$$
\delta G_{i j}=\frac{\mu_{0}^{2}}{8}\left(32+\beta_{3}^{2}\right) \eta_{i j}, \quad \delta G_{z z}=\frac{3 \mu_{0}^{2}}{8}\left(32+\beta_{3}^{2}\right) .
$$

Plugging (4.36) into (4.34), we obtain

$$
-\int_{\mathcal{H}_{0}} d \Sigma^{t} \xi^{t} \delta G_{t t}=-\frac{\pi L^{2}}{48}\left(32+\beta_{3}^{2}\right)\left(\mu_{0} l\right)^{2}+\frac{\pi L^{2}}{128} \frac{l}{z_{\Lambda}}\left(32+\beta_{3}^{2}\right)\left(\mu_{0} l\right)^{2} .
$$

The divergent term in $z_{\Lambda} \rightarrow 0$ limit in (4.37) is cancelled by $\delta \gamma_{A}^{(c t)}$ in (4.34) and the finite variation of the area is equivalent to the $\delta \gamma_{A}$ in (3.32). Finally, we identify the variation of the EE obtained from field theory calculations with the one obtained from the RT formula,

$$
-2 \pi \int_{\mathcal{H}^{+}} d \Sigma^{\mu} \xi^{\nu} \tilde{T}_{\mu \nu}=-\frac{1}{4 G_{N}^{(4)}} \int_{\mathcal{H}_{0}} d \Sigma^{\mu} \xi^{\nu} \delta G_{\mu \nu}
$$

For the Killing vector $\xi^{\mu}$, the term in the left-hand side of (4.38) defines a conserved quantity and thus one can choose any surface homologous to $\mathcal{H}^{+}$as the integration surface. For the choice of the surface at $t=0$, the integration surfaces of both sides in (4.38) are identified. Then, from this relation, we obtain

$$
\delta G_{\mu \nu}=8 \pi G_{N}^{(4)} \tilde{T}_{\mu \nu},
$$

which is the linearized Einstein equation with matter fields. Inserting (4.24) and (4.36) into (4.39), one can determine the numerical factors $A_{\psi}$ and $A_{t}$, which exactly match the values given in (2.55). Therefore, one can see that the RT formula satisfying the Einstein equation reproduces the variation of the EE calculated in the path integral method in the field theory side.

\section{Conclusion}

In this paper, we investigated the phenomena of the emergent gravity in 4-dimensions from the EE of the 3-dimensional mABJM theory. Using the path integral method developed in [32] and the RT formula, we clarified the relation between the emergent (auxiliary) gravity and the Einstein-Hilbert action with two scalar fields in 4-dimensions, which is obtained from the KK reduction of the 11-dimensional supergravity on the LLM geometries with $\mathbb{Z}_{k}$ orbifold.

Our analysis relies heavily on the gauge/gravity duality between the mABJM theory and the 11-dimensional supergravity on the LLM geometries. In order to setup the gauge/gravity dictionary, we need to construct the 4-dimensional gravity using the KK reduction on the compact manifold $S^{7} / \mathbb{Z}_{k}$. In our previous work [26, 27], we showed an exact dual relation for the vev of a CPO of conformal dimension one $\left(\mathcal{O}^{(1)}\right)$ in mABJM theory and a scalar field in an asymptotically $\mathrm{AdS}_{4}$ gravity theory in the large $N$ limit. However, the connection between the 4-dimensional graviton mode and the 11-dimensional 
fluctuations was missing. In this paper, for the minimal ingredients that encode all the information of the LLM geometries with $\mathbb{Z}_{k}$ orbifold in the asymptotic limit up to $\mu_{0^{-}}^{2}$ order, we completed the non-trivial KK maps between the 4-dimensional fields and the 11-dimensional fluctuations on $\mathrm{AdS}_{4} \times S^{7} / \mathbb{Z}_{k}$. The resulting 4-dimensional fields are composed of the graviton mode $H_{\mu \nu}$, one scalar field $\Psi$, and one pseudoscalar field $T$. In the matter sector, the asymptotic behaviour of the scalar field $\Psi$ determines the vev of $\mathcal{O}^{(1)}$ whereas the asymptotic behaviour of the pseudoscalar field $T$ determines the source which couples to a gauge invariant operator of conformal dimension two $\left(\tilde{\mathcal{O}}^{(2)}\right)$.

The presence of these scalar fields in the 4-dimensional gravity theory implies the existence of some relevant deformation in the dual CFT and as a result the EE is expected to show a variation from that of the CFT. Employing the holographic RT formula for subregion $A$, which is a disk of radius $l$, we calculated the leading order contribution to the variation of the $\mathrm{EE} \delta S_{A}$ in mABJM theory by using the 4-dimensional metric $\left(g_{\mu \nu}+H_{\mu \nu}\right)$, which encodes the information of the asymptotic LLM geometries in small mass limit. We showed that, the leading order contribution to the $\delta S_{A}$ is quadratic in the deformation parameter $\mu_{0}$. At such leading order, $\delta S_{A}$ is completely fixed by the vev of $\mathcal{O}^{(1)}$ and the source which couples to $\tilde{\mathcal{O}}^{(2)}$. The obtained $\delta S_{A}$ is negative, which is consistent with the $F$-theorem in 3-dimensional gauge theory and describes the RG flow from UV fixed point of the conformal invariant ABJM theory.

Based on a recent progress in the computation of the EE by using the path integral method in CFT with some relevant deformations, we reproduced the HEE for the mABJM theory. In order to calculate the variation of EE using the path integral method, it is necessary to introduce an additional coordinate $z$ and one auxiliary scalar field for every relevant operator added to the CFT action. In the quadratic order of approximation, the mABJM theory is regarded as a deformation of the ABJM theory by the relevant operators $\mathcal{O}^{(1)}$ and $\tilde{\mathcal{O}}^{(2)}$. Therefore, we need to introduce two auxiliary scalar fields. We identified these auxiliary fields with the scalar fields $\Psi$ and $T$, in the calculation of the EE using the path integral approach. Consistent with the holographic method, the leading order contribution to $\delta S_{A}$ from the path integral approach is quadratic in the deformation parameter. Furthermore, the variation of HEE obtained from the RT formula and the $\delta S_{A}$ from the path integral methods are equal, when the linearized Einstein equation with the energy-momentum tensor of the two scalar fields $\Psi$ and $T$, is satisfied. This Einstein equation agrees with the one we obtained from the KK reduction of the 11-dimensional supergravity on LLM geometries. This agreement and the appearance of the additional coordinate $z$ are the indications of the emergence of an asymptotically $\mathrm{AdS}_{4}$ gravity from the quantum entanglement of the 3-dimensional mABJM theory.

In the calculation of $\delta S_{A}$ in terms of the path integral method developed in [32], we used the energy-momentum tensor for the two scalar fields $\Psi$ and $T$ in the dual 4-dimensional gravity theory, relying on the exact dual relation in our previous work in $[26,27]$. However, in general, one can compute the $\delta S_{A}$ up to $\mu_{0}^{2}$-order in the path integral method by using the mABJM theory directly without using the dual theory. We leave this for future work. 


\section{Acknowledgments}

OK would like to thank the participants of the 5th IBS Brainstrom workshop for stimulating discussions and appreciates APCTP for its hospitality during completion of this work. DT would like to thank the physics department of Addis Ababa University for hospitality, during the visit to present part of this work. This work was supported by the National Research Foundation of Korea(NRF) grant with grant number NRF-2016R1D1A1B03931090 (Y.K.), NRF-2017R1D1A1A09000951 (O.K.), and NRF-2017R1D1A1B03032523 (D.T.).

Open Access. This article is distributed under the terms of the Creative Commons Attribution License (CC-BY 4.0), which permits any use, distribution and reproduction in any medium, provided the original author(s) and source are credited.

\section{References}

[1] S. Ryu and T. Takayanagi, Holographic derivation of entanglement entropy from AdS/CFT, Phys. Rev. Lett. 96 (2006) 181602 [hep-th/0603001] [InSPIRE].

[2] S. Ryu and T. Takayanagi, Aspects of Holographic Entanglement Entropy, JHEP 08 (2006) 045 [hep-th/0605073] [INSPIRE].

[3] V.E. Hubeny, M. Rangamani and T. Takayanagi, A covariant holographic entanglement entropy proposal, JHEP 07 (2007) 062 [arXiv:0705.0016] [INSPIRE].

[4] J.M. Maldacena, The large $N$ limit of superconformal field theories and supergravity, Int. J. Theor. Phys. 38 (1999) 1113 [hep-th/9711200] [INSPIRE].

[5] S.S. Gubser, I.R. Klebanov and A.M. Polyakov, Gauge theory correlators from noncritical string theory, Phys. Lett. B 428 (1998) 105 [hep-th/9802109] [InSPIRE].

[6] E. Witten, Anti-de Sitter space and holography, Adv. Theor. Math. Phys. 2 (1998) 253 [hep-th/9802150] [INSPIRE].

[7] B. Swingle, Entanglement Renormalization and Holography, Phys. Rev. D 86 (2012) 065007 [arXiv: 0905.1317] [INSPIRE].

[8] M. Van Raamsdonk, Comments on quantum gravity and entanglement, arXiv:0907.2939 [INSPIRE].

[9] M. Van Raamsdonk, Building up spacetime with quantum entanglement, Gen. Rel. Grav. 42 (2010) 2323 [arXiv:1005.3035] [INSPIRE].

[10] J. Bhattacharya, M. Nozaki, T. Takayanagi and T. Ugajin, Thermodynamical Property of Entanglement Entropy for Excited States, Phys. Rev. Lett. 110 (2013) 091602 [arXiv: 1212.1164] [INSPIRE].

[11] D. Allahbakhshi, M. Alishahiha and A. Naseh, Entanglement Thermodynamics, JHEP 08 (2013) 102 [arXiv:1305.2728] [INSPIRE].

[12] D.D. Blanco, H. Casini, L.-Y. Hung and R.C. Myers, Relative Entropy and Holography, JHEP 08 (2013) 060 [arXiv:1305.3182] [INSPIRE].

[13] G. Wong, I. Klich, L.A. Pando Zayas and D. Vaman, Entanglement Temperature and Entanglement Entropy of Excited States, JHEP 12 (2013) 020 [arXiv: 1305.3291] [INSPIRE]. 
[14] N. Lashkari, M.B. McDermott and M. Van Raamsdonk, Gravitational dynamics from entanglement 'thermodynamics', JHEP 04 (2014) 195 [arXiv: 1308.3716] [INSPIRE].

[15] T. Faulkner, M. Guica, T. Hartman, R.C. Myers and M. Van Raamsdonk, Gravitation from Entanglement in Holographic CFTs, JHEP 03 (2014) 051 [arXiv:1312.7856] [INSPIRE].

[16] B. Swingle and M. Van Raamsdonk, Universality of Gravity from Entanglement, arXiv: 1405.2933 [INSPIRE].

[17] J.-W. Lee, H.-C. Kim and J. Lee, Gravity from Quantum Information, J. Korean Phys. Soc. 63 (2013) 1094 [arXiv: 1001.5445] [INSPIRE].

[18] B. Mosk, Holographic equivalence between the first law of entanglement entropy and the linearized gravitational equations, Phys. Rev. D 94 (2016) 126001 [arXiv:1608.06292] [INSPIRE].

[19] B. Czech, L. Lamprou, S. McCandlish, B. Mosk and J. Sully, Equivalent Equations of Motion for Gravity and Entropy, JHEP 02 (2017) 004 [arXiv: 1608.06282] [INSPIRE].

[20] E. Oh, I.Y. Park and S.-J. Sin, Complete Einstein equation from the generalized First Law of Entanglement, arXiv:1709.05752 [INSPIRE].

[21] T. Faulkner, F.M. Haehl, E. Hijano, O. Parrikar, C. Rabideau and M. Van Raamsdonk, Nonlinear Gravity from Entanglement in Conformal Field Theories, JHEP 08 (2017) 057 [arXiv: 1705.03026] [INSPIRE].

[22] K. Hosomichi, K.-M. Lee, S. Lee, S. Lee and J. Park, $N=5,6$ Superconformal Chern-Simons Theories and M2-branes on Orbifolds, JHEP 09 (2008) 002 [arXiv:0806.4977] [INSPIRE].

[23] J. Gomis, D. Rodriguez-Gomez, M. Van Raamsdonk and H. Verlinde, A Massive Study of M2-brane Proposals, JHEP 09 (2008) 113 [arXiv:0807.1074] [INSPIRE].

[24] O. Aharony, O. Bergman, D.L. Jafferis and J. Maldacena, $N=6$ superconformal Chern-Simons-matter theories, M2-branes and their gravity duals, JHEP 10 (2008) 091 [arXiv:0806.1218] [INSPIRE].

[25] H. Lin, O. Lunin and J.M. Maldacena, Bubbling AdS space and 1/2 BPS geometries, JHEP 10 (2004) 025 [hep-th/0409174] [INSPIRE].

[26] D. Jang, Y. Kim, O.-K. Kwon and D.D. Tolla, Exact Holography of the Mass-deformed M2-brane Theory, Eur. Phys. J. C 77 (2017) 342 [arXiv:1610.01490] [INSPIRE].

[27] D. Jang, Y. Kim, O.-K. Kwon and D.D. Tolla, Mass-deformed ABJM Theory and LLM Geometries: Exact Holography, JHEP 04 (2017) 104 [arXiv: 1612.05066] [INSPIRE].

[28] K. Skenderis and M. Taylor, Kaluza-Klein holography, JHEP 05 (2006) 057 [hep-th/0603016] [INSPIRE].

[29] K. Skenderis and M. Taylor, Holographic Coulomb branch vevs, JHEP 08 (2006) 001 [hep-th/0604169] [INSPIRE].

[30] K. Skenderis and M. Taylor, Anatomy of bubbling solutions, JHEP 09 (2007) 019 [arXiv: 0706.0216] [INSPIRE].

[31] D. Jang, Y. Kim, O.-K. Kwon and D.D. Tolla, Holography of Massive M2-brane Theory: Non-linear Extension, arXiv:1803.10660 [INSPIRE].

[32] T. Faulkner, Bulk Emergence and the RG Flow of Entanglement Entropy, JHEP 05 (2015) 033 [arXiv:1412.5648] [INSPIRE]. 
[33] V. Rosenhaus and M. Smolkin, Entanglement Entropy: A Perturbative Calculation, JHEP 12 (2014) 179 [arXiv: 1403.3733] [INSPIRE].

[34] V. Rosenhaus and M. Smolkin, Entanglement Entropy for Relevant and Geometric Perturbations, JHEP 02 (2015) 015 [arXiv: 1410.6530] [INSPIRE].

[35] T. Faulkner, R.G. Leigh and O. Parrikar, Shape Dependence of Entanglement Entropy in Conformal Field Theories, JHEP 04 (2016) 088 [arXiv: 1511.05179] [INSPIRE].

[36] F.M. Haehl, Comments on universal properties of entanglement entropy and bulk reconstruction, JHEP 10 (2015) 159 [arXiv:1508.00766] [INSPIRE].

[37] A.J. Speranza, Entanglement entropy of excited states in conformal perturbation theory and the Einstein equation, JHEP 04 (2016) 105 [arXiv: 1602.01380] [INSPIRE].

[38] M.J.S. Beach, J. Lee, C. Rabideau and M. Van Raamsdonk, Entanglement entropy from one-point functions in holographic states, JHEP 06 (2016) 085 [arXiv:1604.05308] [INSPIRE].

[39] G. Sárosi and T. Ugajin, Modular Hamiltonians of excited states, OPE blocks and emergent bulk fields, JHEP 01 (2018) 012 [arXiv: 1705.01486] [INSPIRE].

[40] H. Liu and M. Mezei, A Refinement of entanglement entropy and the number of degrees of freedom, JHEP 04 (2013) 162 [arXiv:1202.2070] [INSPIRE].

[41] M. Nozaki, T. Numasawa, A. Prudenziati and T. Takayanagi, Dynamics of Entanglement Entropy from Einstein Equation, Phys. Rev. D 88 (2013) 026012 [arXiv:1304.7100] [INSPIRE].

[42] J. Lin, M. Marcolli, H. Ooguri and B. Stoica, Locality of Gravitational Systems from Entanglement of Conformal Field Theories, Phys. Rev. Lett. 114 (2015) 221601 [arXiv: 1412.1879] [INSPIRE].

[43] N. Lashkari and M. Van Raamsdonk, Canonical Energy is Quantum Fisher Information, JHEP 04 (2016) 153 [arXiv: 1508.00897] [INSPIRE].

[44] H. Casini, D.A. Galante and R.C. Myers, Comments on Jacobson's "entanglement equilibrium and the Einstein equation", JHEP 03 (2016) 194 [arXiv:1601.00528] [INSPIRE].

[45] M. Taylor and W. Woodhead, Renormalized entanglement entropy, JHEP 08 (2016) 165 [arXiv: 1604.06808] [INSPIRE].

[46] C. Kim, K.K. Kim and O.-K. Kwon, Holographic Entanglement Entropy of Anisotropic Minimal Surfaces in LLM Geometries, Phys. Lett. B 759 (2016) 395 [arXiv:1605.00849] [INSPIRE].

[47] K.K. Kim, O.-K. Kwon, C. Park and H. Shin, Renormalized Entanglement Entropy Flow in Mass-deformed ABJM Theory, Phys. Rev. D 90 (2014) 046006 [arXiv:1404.1044] [INSPIRE].

[48] K.K. Kim, O.-K. Kwon, C. Park and H. Shin, Holographic entanglement entropy of mass-deformed Aharony-Bergman-Jafferis-Maldacena theory, Phys. Rev. D 90 (2014) 126003 [arXiv: 1407.6511] [INSPIRE].

[49] R. Auzzi and S.P. Kumar, Non-Abelian Vortices at Weak and Strong Coupling in Mass Deformed ABJM Theory, JHEP 10 (2009) 071 [arXiv:0906.2366] [INSPIRE].

[50] S. Cheon, H.-C. Kim and S. Kim, Holography of mass-deformed M2-branes, arXiv:1101.1101 [INSPIRE]. 
[51] M. Cvetič, H. Lü and C.N. Pope, Consistent Kaluza-Klein sphere reductions, Phys. Rev. D 62 (2000) 064028 [hep-th/0003286] [InSPIRE].

[52] H.-C. Kim and S. Kim, Supersymmetric vacua of mass-deformed M2-brane theory, Nucl. Phys. B 839 (2010) 96 [arXiv:1001.3153] [InSPIRE].

[53] D. Bak, K.K. Kim and S. Yun, Symmetry Breaking Phase Transitions in ABJM Theory with a Finite U(1) Chemical Potential, Phys. Rev. D 84 (2011) 086010 [arXiv:1011.5749] [INSPIRE].

[54] I. Bena, The M-theory dual of a three-dimensional theory with reduced supersymmetry, Phys. Rev. D 62 (2000) 126006 [hep-th/0004142] [INSPIRE].

[55] N. Lashkari, C. Rabideau, P. Sabella-Garnier and M. Van Raamsdonk, Inviolable energy conditions from entanglement inequalities, JHEP 06 (2015) 067 [arXiv:1412.3514] [INSPIRE].

[56] D.L. Jafferis, A. Lewkowycz, J. Maldacena and S.J. Suh, Relative entropy equals bulk relative entropy, JHEP 06 (2016) 004 [arXiv: 1512.06431] [INSPIRE].

[57] B. Ning and F.-L. Lin, Relative Entropy and Torsion Coupling, Phys. Rev. D 94 (2016) 126007 [arXiv: 1607.00263] [INSPIRE].

[58] S. Hollands and R.M. Wald, Stability of Black Holes and Black Branes, Commun. Math. Phys. 321 (2013) 629 [arXiv:1201.0463] [INSPIRE].

[59] S. Hyun, S.-A. Park and S.-H. Yi, Canonical energy and hairy AdS black holes, Phys. Rev. D 94 (2016) 044014 [arXiv: 1603.02530] [InSPIRE].

[60] S. Banerjee, J. Erdmenger and D. Sarkar, Connecting Fisher information to bulk entanglement in holography, arXiv:1701.02319 [INSPIRE].

[61] H. Casini, M. Huerta and R.C. Myers, Towards a derivation of holographic entanglement entropy, JHEP 05 (2011) 036 [arXiv:1102.0440] [INSPIRE].

[62] V. Balasubramanian and P. Kraus, A Stress tensor for Anti-de Sitter gravity, Commun. Math. Phys. 208 (1999) 413 [hep-th/9902121] [INSPIRE].

[63] S. de Haro, S.N. Solodukhin and K. Skenderis, Holographic reconstruction of space-time and renormalization in the AdS/CFT correspondence, Commun. Math. Phys. 217 (2001) 595 [hep-th/0002230] [INSPIRE].

[64] K. Skenderis, Asymptotically Anti-de Sitter space-times and their stress energy tensor, Int. J. Mod. Phys. A 16 (2001) 740 [hep-th/0010138] [INSPIRE].

[65] M. Bianchi, D.Z. Freedman and K. Skenderis, Holographic renormalization, Nucl. Phys. B 631 (2002) 159 [hep-th/0112119] [INSPIRE].

[66] R.M. Wald, Black hole entropy is the Noether charge, Phys. Rev. D 48 (1993) R3427 [gr-qc/9307038] [INSPIRE].

[67] V. Iyer and R.M. Wald, Some properties of Noether charge and a proposal for dynamical black hole entropy, Phys. Rev. D 50 (1994) 846 [gr-qc/9403028] [INSPIRE]. 